\title{
Nonlinear Elliptic Equations with Singular Terms and Combined Nonlinearities
}

\author{
Leszek Gasiński and Nikolaos S. Papageorgiou
}

\begin{abstract}
We consider nonlinear elliptic Dirichlet problems with a singular term, a concave (i.e., $(p-1)$-sublinear) term and a Carathéodory perturbation. We study the cases where the Carathéodory perturbation is $(p-1)$-linear and $(p-1)$-superlinear near $+\infty$. Using variational techniques based on the critical point theory together with truncation arguments and the method of upper and lower solutions, we show that if the $L^{\infty}$-coefficient of the concave term is small enough, the problem has at least two nontrivial smooth solutions.
\end{abstract}

\section{Introduction}

Let $\Omega \subseteq \mathbb{R}^{N}$ be a bounded domain with a $C^{2}$-boundary $\partial \Omega$. In this paper we study the following nonlinear Dirichlet problem:

$$
\left\{\begin{array}{l}
-\Delta_{p} u(z)=a(z) u(z)^{-\eta}+m(z) u(z)^{q-1}+f(z, u(z)) \quad \text { in } \Omega, \\
\left.u\right|_{\partial \Omega}=0, u>0
\end{array}\right.
$$

with $1<q<p, \eta>0$. Here $\Delta_{p}$ denotes the $p$-Laplacian differential operator, defined by

$$
\Delta_{p} u=\operatorname{div}\left(\|\nabla u\|^{p-2} \nabla u\right) \quad \forall u \in W_{0}^{1, p}(\Omega),
$$

with $p \in(1,+\infty)$.

We observe that problem (1.1) has a singular term $a(\cdot) u(\cdot)^{-\eta}$ and a $(p-1)$-sublinear term (concave term) $m(\cdot) u(\cdot)^{q-1}, 1<q<p$. The function $f$ is Carathéodory and concerning its growth properties, we consider two distinct

This research has been partially supported by the Ministry of Science and Higher Education of Poland under Grant no. N201 542438. 
cases. First, we assume that for almost all $z \in \Omega, f(z, \cdot)$ is $(p-1)$-linear near $+\infty$. Subsequently, we examine the case where $f(z, \cdot)$ exhibits a $(p-1)$-superlinear growth near $+\infty$. So, in this second case in problem (1.1) we have the combined effects of concave and convex terms.

Problems with singular terms were studied primarily in the context of semilinear equations (i.e., $p=2$ ). In this connection, we mention the works of Coclite and Palmieri [7], Egnell [11], Hsu and Lin [22], Lair and Shaker [23], Shi and Yao [29], Sun et al. [30], Terracini [31], Zhang [33]. From the aforementioned works, Egnell [11], Hsu and Lin [22] and Terracini [31] consider perturbations with critical growth. In addition, Hsu and Lin [22] have also a concave term of the form

$$
\lambda g(z) u(z)^{q-1}, \quad 1 \leqslant q<2, \quad \lambda>0 .
$$

In these works, the singularity has the form of an inverse square coefficient. Coclite and Palmieri [7] investigate the case of superlinear and subcritical perturbation. More precisely, they assume that

$$
f(z, \zeta)=f(\zeta)=\lambda \zeta^{r-1}
$$

with

$$
2<r<2^{*}= \begin{cases}\frac{2 N}{N-2} & \text { if } N>2, \\ +\infty & \text { if } N=1,2\end{cases}
$$

with $\lambda>0$ being a parameter. They do not have a concave term (i.e., $m \equiv 0$ ). Shi and Yao [29] consider the complementary case, namely they have a sublinear perturbation of the form

$$
f(z, \zeta)=f(\zeta)=\lambda \zeta^{r-1}, \quad 1<r \leqslant 2 .
$$

In both papers, the authors show that there exists $\lambda^{*}>0$, such that for every $\lambda \in\left(0, \lambda^{*}\right)$ the problem admits a nontrivial positive solution. Lair and Shaker [23] have

$$
f \equiv m=0 \quad \text { and } \quad a \in L^{2}(\Omega)
$$

and they establish the existence of a unique positive weak solution. Sun et al. [30] use the Ekeland variational principle to produce two positive solutions for certain superlinear perturbations with $m \equiv 0$ (no concave term). Finally, Zhang [33] extended the work of Sun et al. [30] to more general nonnegative superlinear perturbations using the critical point theory on closed convex sets. Recently, there appeared some works dealing with singular equations driven by $p$-Laplacian. In this connection, we mention the papers of Agarwal et al. [1,3], Agarwal and O'Regan [2], Giacomoni et al. [19], Giacomoni and Saoudi [18], Hernández and Mancebo [21], Perera and Silva [27], Perera and Zhang [28]. In Agarwal et al. [1] and Agarwal and O'Regan [2] the authors consider ordinary differential equations (i.e., $N=1$ ). Agarwal et al. [3] consider the nonresonant coercive case with $m \equiv 0$ and prove an existence theorem. Perera and Silva [27] and Perera and Zhang [28] consider parametric problems with $m \equiv 0$ (i.e., the perturbation of the singular term has the form $\lambda f(z, \zeta), \lambda>0$ ); 
they assume that $f(z, \cdot)$ is $(p-1)$-superlinear and more precisely that it satisfies the well-known Ambrosetti-Rabinowitz condition. They show that there exists $\lambda^{*}>0$, such that for all $\lambda \in\left(0, \lambda^{*}\right)$ the parametric problem has two positive weak solutions. In Perera and Zhang [28] it is assumed that $p \geqslant 2$. In Giacomoni et al. [19] the authors have a singular term of the form $\frac{1}{\zeta^{\delta}}$ with $\delta \in(0,1]$ and a $p$-superlinear perturbation of the form

$$
f(z, \zeta)=f(\zeta)=\zeta^{q},
$$

with $p<q<p^{*}-1$. They prove a result relating $C^{1}(\Omega)$ and $W_{0}^{1, p}(\Omega)$-minimizers and also have multiplicity results. In Giacomoni and Saoudi [18], the authors consider more general singular terms and also a more general $p$-superlinear perturbation, permitting also critical growth. They focus on the relation between $C^{1}$ and Sobolev minimizers and present a simpler proof based on an $L^{p}$-minimization (an approach already present in the work of Brock et al. [6]). We also mention the work of Giacomoni et al. [17] who have a similar result for critical problems in $\mathbb{R}^{N}$. In Hernández and Mancebo [21] we find a comprehensive overview of singular elliptic and parabolic equations. To the best of our knowledge, there are no works in the literature dealing with $p$-Laplacian equations with singular terms and combined nonlinearities (i.e., with the combined effects of concave and convex terms or of concave and linear terms). For equations without singular terms, such problems were investigated by Ambrosetti et al. [4] and Li et al. [24] (semilinear case, i.e., $p=2$ ) and by de Figueiredo et al. [9], García Azorero et al. [13], Guo and Zhang [20], de Paiva and Massa [10], Perera [26] (nonlinear case, i.e., $p>1$ ).

Our approach is variational based on the critical point theory and we also use truncation techniques and the method of upper and lower solutions. In the next section, for the convenience of the reader, we briefly recall the mathematical tools we use in the study of problem (1.1).

\section{Mathematical Background}

Let $X$ be a Banach space and $X^{*}$ its topological dual. By $\langle\cdot, \cdot\rangle$ we denote the duality brackets for the pair $\left(X^{*}, X\right)$. Let $\varphi \in C^{1}(X)$. We say that $\varphi$ satisfies the Cerami condition at the level $c \in \mathbb{R}$ (the $C_{c}$-condition, for short), if the following is true: "Every sequence $\left\{x_{n}\right\}_{n \geqslant 1} \subseteq X$, such that

$$
\varphi\left(x_{n}\right) \longrightarrow c \text { in } \mathbb{R}
$$

and

$$
\left(1+\left\|x_{n}\right\|\right) \varphi^{\prime}\left(x_{n}\right) \longrightarrow 0 \text { in } X^{*},
$$

has a strongly convergent subsequence." We say that $\varphi$ satisfies the Cerami condition (the $C$-condition, for short), if it satisfies the $C_{c^{-}}$-condition at every level $c \in \mathbb{R}$.

This compactness type condition on $\varphi$ is weaker than the usual PalaisSmale condition. However, as it was shown in Bartolo et al. [5], the deformation theorem and consequently the minimax theory of the critical values of $\varphi$ 
is still valid if the Palais-Smale condition is replaced by the Cerami condition. In particular, we have the following form of the well-known "mountain pass theorem" (see e.g., Gasiński and Papageorgiou [14, p. 140, Theorem 2.1.3]).

Theorem 2.1. If $X$ is a Banach space, $\varphi \in C^{1}(X), x_{0}, x_{1} \in X, \varrho>0$, $\left\|x_{1}-x_{0}\right\|>\varrho$,

$$
\begin{aligned}
\max \left\{\varphi\left(x_{0}\right), \varphi\left(x_{1}\right)\right\} & \leqslant \inf \left\{\varphi(x):\left\|x-x_{0}\right\|=\varrho\right\}=\eta, \\
c & =\inf _{\gamma \in \Gamma} \max _{0 \leqslant t \leqslant 1} \varphi(\gamma(t)),
\end{aligned}
$$

where

$$
\Gamma=\left\{\gamma \in C([0,1] ; X): \gamma(0)=x_{0}, \gamma(1)=x_{1}\right\}
$$

and $\varphi$ satisfies the $C_{c}$-condition, then $c \geqslant \eta$ and $c$ is a critical value of $\varphi$. Moreover, if $c=\eta$, then there exists a critical point $x \in X$ of $\varphi$ with $\varphi(x)=c$ and $\left\|x-x_{0}\right\|=\varrho$.

The notion of a map of $(S)_{+}$type is useful in proving that a $C^{1}$-functional satisfies the $C$-condition. We say that a map $A: X \longrightarrow X^{*}$ is of type $(S)_{+}$, if for every sequence $\left\{x_{n}\right\}_{n \geqslant 1} \subseteq X$, such that

$$
x_{n} \longrightarrow x \text { weakly in } X \text { and } \limsup _{n \rightarrow+\infty}\left\langle A\left(x_{n}\right), x_{n}-x\right\rangle \leqslant 0,
$$

we have

$$
x_{n} \longrightarrow x \text { in } X .
$$

Let $X=W_{0}^{1, p}(\Omega)$ and $X^{*}=W^{-1, p^{\prime}}(\Omega)$ (where $\frac{1}{p}+\frac{1}{p^{\prime}}=1$ ). We consider the map $A: W_{0}^{1, p}(\Omega) \longrightarrow W^{-1, p^{\prime}}(\Omega)$ corresponding to the $p$-Laplacian, defined by

$$
\langle A(u), y\rangle=\int_{\Omega}\|\nabla u\|^{p-2}(\nabla u, \nabla y)_{\mathbb{R}^{N}} \mathrm{~d} z \quad \forall u, y \in W_{0}^{1, p}(\Omega)
$$

We have the following properties of $A$ (see Gasiński and Papageorgiou $[15])$ :

Proposition 2.2. The map $A: W_{0}^{1, p}(\Omega) \longrightarrow W^{-1, p^{\prime}}(\Omega)$ defined by $(2.1)$ is of type $(S)_{+}$.

As we have already mentioned, our approach uses the method of upper and lower solutions. So, next we recall the definitions of upper and lower solutions for problem (1.1).

Definition 2.3. (a) We say that $\bar{u} \in W^{1, p}(\Omega)$ is an upper solution for problem (1.1), if

$$
\left\{\begin{array}{l}
\int_{\Omega}\|\nabla \bar{u}\|^{p-2}(\nabla \bar{u}, \nabla h)_{\mathbb{R}^{N}} \mathrm{~d} z \geqslant \int_{\Omega} a \bar{u}^{-\eta} h \mathrm{~d} z+\int_{\Omega} m \bar{u}^{q-1} \mathrm{~d} z+\int_{\Omega} f(z, \bar{u}) h \mathrm{~d} z \\
\forall h \in C_{c}^{1}(\bar{\Omega}), h \geqslant 0, \\
\left.\bar{u}\right|_{\partial \Omega} \geqslant 0 .
\end{array}\right.
$$

We say that $\bar{u}$ is a strict upper solution, if it is an upper solution for (1.1) but not a solution. 
(b) We say that $\underline{u} \in W^{1, p}(\Omega)$ is a lower solution for problem (1.1), if

$$
\left\{\begin{aligned}
\int_{\Omega} \| & \nabla \underline{u} \|^{p-2}(\nabla \underline{u}, \nabla h)_{\mathbb{R}^{N}} \mathrm{~d} z \leqslant \int_{\Omega} a \underline{u}^{-\eta} h \mathrm{~d} z+\int_{\Omega} m \underline{u}^{q-1} \mathrm{~d} z+\int_{\Omega} f(z, \underline{u}) h \mathrm{~d} z \\
& \forall h \in C_{c}^{1}(\bar{\Omega}), h \geqslant 0, \\
& \left.\underline{u}\right|_{\partial \Omega} \leqslant 0 .
\end{aligned}\right.
$$

We say that $\underline{u}$ is a strict lower solution, if it is a lower solution for (1.1) but not a solution.

In the study of problem (1.1), in addition to the Sobolev space $W_{0}^{1, p}(\Omega)$ we will also use the space

$$
C_{0}^{1}(\bar{\Omega})=\left\{u \in C^{1}(\bar{\Omega}):\left.u\right|_{\partial \Omega}=0\right\} .
$$

This is an ordered Banach space with positive cone

$$
C_{+}=\left\{u \in C_{0}^{1}(\bar{\Omega}): u(z) \geqslant 0 \text { for all } z \in \bar{\Omega}\right\} .
$$

This cone has a nonempty interior, given by

$$
\operatorname{int} C_{+}=\left\{u \in C_{+}: u(z)>0 \text { for all } z \in \Omega, \frac{\partial u}{\partial n}(z)<0 \text { for all } z \in \partial \Omega\right\},
$$

(here $n(\cdot)$ denotes the outward unit normal on $\partial \Omega$ ).

The next result will be helpful in our arguments and its proof can be found in Perera and Zhang [28].

Theorem 2.4. If $h \in L^{s}(\Omega)_{+} \backslash\{0\}$ with $N<s \leqslant+\infty$, then the Dirichlet problem

$$
\left\{\begin{array}{l}
-\Delta_{p} u(z)=h(z) \quad \text { in } \Omega \\
\left.u\right|_{\partial \Omega}=0
\end{array}\right.
$$

has a unique strong solution $u \in \operatorname{int} C_{+}$.

Finally, let us briefly recall some basic facts about the spectrum of the negative Dirichlet $p$-Laplacian (denoted by $\left(-\Delta_{p}, W_{0}^{1, p}(\Omega)\right)$ ). So, we consider the following nonlinear eigenvalue problem:

$$
\left\{\begin{array}{l}
-\Delta_{p} u(z)=\lambda|u(z)|^{p-2} u(z) \quad \text { in } \Omega \\
\left.u\right|_{\partial \Omega}=0
\end{array}\right.
$$

A number $\lambda \in \mathbb{R}$ for which problem (2.2) has a nontrivial solution is said to be an eigenvalue of $\left(-\Delta_{p}, W_{0}^{1, p}(\Omega)\right)$. The smallest eigenvalue $\lambda_{1}$ of $\left(-\Delta_{p}, W_{0}^{1, p}(\Omega)\right)$ is positive, isolated, simple and admits the following variational characterization:

$$
\lambda_{1}=\inf \left\{\frac{\|\nabla u\|_{p}^{p}}{\|u\|_{p}^{p}}: u \in W_{0}^{1, p}(\Omega), u \neq 0\right\} .
$$

The infimum in (2.3) is attained on the corresponding one-dimensional eigenspace. In what follows, by $\widehat{u}_{1}$ we denote the $L^{p}$-normalized (i.e., $\left\|\widehat{u}_{1}\right\|_{p}=1$ ) eigenfunction corresponding to $\lambda_{1}>0$. From (2.3) it is clear that $\widehat{u}_{1}$ does not change sign and so we set $\widehat{u}_{1} \geqslant 0$. The nonlinear regularity theory (see e.g., 
Gasiński and Papageorgiou [15, pp. 737-738]) implies that $\widehat{u}_{1} \in C_{+}$. In fact the nonlinear maximum principle of Vázquez [32] implies that $\widehat{u}_{1} \in \operatorname{int} C_{+}$.

The Ljusternik-Schnirelmann theory provides a whole sequence $\left\{\lambda_{n}\right\}_{n} \geqslant 1$ of strictly increasing eigenvalues of $\left(-\Delta_{p}, W_{0}^{1, p}(\Omega)\right)$, such that $\lambda_{n} \rightarrow+\infty$. These eigenvalues are known as the Ljusternik-Schnirelmann eigenvalues. If $p=2$ (linear eigenvalue problem), then these are all the eigenvalues of $\left(-\Delta, H_{0}^{1}(\Omega)\right)$. If $p \neq 2$ (nonlinear eigenvalue problem) we do not know if this is the case. However, since $\lambda_{1}>0$ is isolated, we can define

$$
\lambda_{2}^{*}=\inf \left\{\lambda: \lambda \text { is an eigenvalue of }\left(-\Delta_{p}, W_{0}^{1, p}(\Omega)\right), \lambda>\lambda_{1}\right\}>\lambda_{1} .
$$

Because the set $\sigma(p)$ of eigenvalues of $\left(-\Delta_{p}, W_{0}^{1, p}(\Omega)\right)$ is closed, $\lambda_{2}^{*}>0$ is an eigenvalue, the second eigenvalue of $\left(-\Delta_{p}, W_{0}^{1, p}(\Omega)\right)$. We have $\lambda_{2}^{*}=\lambda_{2}$, i.e., the second Ljusternik-Schnirelmann eigenvalue and the second eigenvalue coincide.

In what follows, we use the notation $r^{ \pm}=\max \{ \pm r, 0\}$ for all $r \in \mathbb{R}$. By $\|\cdot\|_{r}$ we denote the norm of $L^{r}(\Omega)$ and by $\|\cdot\|$ the norm of the Sobolev space $W_{0}^{1, p}(\Omega)$ or of $\mathbb{R}^{N}$ - it will always be clear from the context. Finally, by $p^{*}$ we denote the critical Sobolev exponent, defined by

$$
p^{*}= \begin{cases}\frac{N p}{N-p} & \text { if } N>p \\ +\infty & \text { if } N \leqslant p .\end{cases}
$$

\section{3. $(p-1)$-Linear Perturbations}

In this section we consider the case where the Carathéodory perturbation $f(z, \zeta)$ exhibits a $(p-1)$-linear growth near $+\infty$. More precisely, the hypotheses on $f$ are the following:

$\underline{H(f)_{1}} f: \Omega \times \mathbb{R} \longrightarrow \mathbb{R}$ is a function, such that

(i) for all $\zeta \in \mathbb{R}$, the function $z \longmapsto f(z, \zeta)$ is measurable;

(ii) for almost all $z \in \Omega$, the function $\zeta \longmapsto f(z, \zeta)$ is continuous and we have $f(z, 0)=0$

(iii) for almost all $z \in \Omega$ and all $\zeta \in \mathbb{R}$, we have

$$
|f(z, \zeta)| \leqslant a(z)+c|\zeta|^{p-1},
$$

with $a \in L^{\infty}(\Omega)_{+}, c>0$;

(iv) if $F(z, \zeta)=\int_{0}^{\zeta} f(z, s) \mathrm{d} s$, then there exist $\vartheta_{0} \in\left(\lambda_{1}, \lambda_{2}\right), \beta_{0}>0$ and $\tau \in[1, p]$, such that

$$
\lambda_{1} \leqslant \liminf _{\zeta \rightarrow+\infty} \frac{p F(z, \zeta)}{\zeta^{p}} \leqslant \limsup _{\zeta \rightarrow+\infty} \frac{p F(z, \zeta)}{\zeta^{p}} \leqslant \vartheta_{0},
$$

uniformly for almost all $z \in \Omega$ and

$$
\liminf _{\zeta \rightarrow+\infty} \frac{p F(z, \zeta)-f(z, \zeta) \zeta}{\zeta^{\tau}} \geqslant \beta_{0}
$$

uniformly for almost all $z \in \Omega$; 
(v) there exists $\vartheta \in L^{\infty}(\Omega)_{+}$, such that $\vartheta(z) \leqslant \lambda_{1}$ for almost all $z \in \Omega$, with the strict inequality on a set of positive measure, and

$$
\liminf _{\zeta \rightarrow 0^{+}} \frac{p F(z, \zeta)}{\zeta^{p}} \leqslant \vartheta(z)
$$

uniformly for almost all $z \in \Omega$ and

$$
m(z) \zeta^{q-1}+f(z, \zeta) \geqslant 0 \text { for almost all } z \in \Omega \text { and all } \zeta \geqslant 0 .
$$

Remark 3.1. Since we are interested in positive solutions and the asymptotic conditions (hypotheses $H(f)_{1}(i v)$ and $(v)$ ) concern only the positive semiaxis, we may (and will) assume that

$$
f(z, \zeta)=0 \quad \text { for almost all } z \in \Omega \text { and all } \zeta \leqslant 0 .
$$

Hypothesis $H(f)_{1}(i v)$ implies that for almost all $z \in \Omega$, the potential function $F(z, \cdot)$ is $p$-linear near $+\infty$. In particular, this is the case if for almost all $z \in \Omega, f(z, \cdot)$ is $(p-1)$-linear near $+\infty$. Note that hypothesis $H(f)_{1}($ iv $)$ permits resonance at $+\infty$ with respect to $\lambda_{1}$. For this reason we need additional conditions on the asymptotic behaviour at $+\infty$ (see the second part of hypothesis $\left.H(f)_{1}(\mathrm{iv})\right)$.

Example 3.2. The following function $f$ satisfies hypotheses $H(f)_{1}$ (for the sake of simplicity we drop the $z$-dependence):

$$
f(\zeta)= \begin{cases}0 & \text { if } \zeta \leqslant 0, \\ \beta \zeta^{p-1}+c \zeta^{\mu-1} & \text { if } 0<\zeta<1, \\ \xi \zeta^{p-1}+\zeta^{\vartheta-1} & \text { if } 1 \leqslant \zeta,\end{cases}
$$

with $\xi \in\left[\lambda_{1}, \lambda_{2}\right), \beta<\lambda_{1}, 1<\vartheta<p<\mu$ and $c=\xi+1-\beta$.

The hypothesis on the singular term are the following:

$H(\eta, a)_{1} \eta>0, a(z) \geqslant 0$ for almost all $z \in \Omega$ and there exist $\widehat{v} \in C_{+}$and $s>N$, such that $a \widehat{v}^{-\eta} \in L^{s}(\Omega)$.

Remark 3.3. In particular, this hypothesis implies that $a \in L^{s}(\Omega)_{+}$(see the proof of Lemma 3.4 below). Note that we do not require that $\eta<1$, a restriction that appears often in the literature (see, e.g., Lair and Shaker [23], Sun et al. [30], Zhang [33]).

Finally for the coefficient $m(z)$ of the concave term, we assume the following:

$H(m) m \in L^{\infty}(\Omega)_{+}, m \neq 0$.

We will generate lower and upper solutions for problem (1.1). First we produce a lower solution:

Lemma 3.4. If hypotheses $H(f)_{1}, H(\eta, a)_{1}$ and $H(m)$ hold, then problem (1.1) admits a lower solution: $\underline{u} \in$ int $C_{+}$. 
Proof. Note that by virtue of hypothesis $H(\eta, a)_{1}$, we can find $M>0$, such that

$$
\frac{a(z)}{M^{\eta}} \leqslant \frac{a(z)}{\widehat{v}(z)^{\eta}}=a(z) \widehat{v}(z)^{-\eta} \quad \text { for almost all } z \in \Omega,
$$

so $a \in L^{s}(\Omega)_{+}\left(\right.$since $\left.a \widehat{v}^{-\eta} \in L^{s}(\Omega)_{+}\right)$.

We consider the following auxiliary Dirichlet problem:

$$
\left\{\begin{array}{l}
-\Delta_{p} v(z)=m(z) a(z) \quad \text { in } \Omega \\
\left.v\right|_{\partial \Omega}=0
\end{array}\right.
$$

By Theorem 2.4 this problem has a unique strong solution $\underline{v} \in \operatorname{int} C_{+}$. Choose $t \in\left(0,\left(\frac{1}{\|m\|_{\infty}}\right)^{\frac{1}{p-1}}\right)$ small enough, such that

$$
\underline{u}(z)=t \underline{v}(z) \in[0,1) \quad \forall z \in \bar{\Omega} .
$$

Then, using (3.1), the facts that $t \in\left(0,\left(\frac{1}{\|m\|_{\infty}}\right)^{\frac{1}{p-1}}\right), a \geqslant 0, \underline{u}(z) \in(0,1)$ for all $z \in \Omega$ and hypothesis $H(f)_{1}(v)$, we have

$$
\begin{aligned}
-\Delta_{p} \underline{u}(z) & =t^{p-1} m(z) a(z) \leqslant a(z) \leqslant a(z) \underline{u}(z)^{-\eta} \\
& \leqslant a(z) \underline{u}(z)^{-\eta}+m(z) \underline{u}(z)^{q-1}+f(z, \underline{u}(z)),
\end{aligned}
$$

for almost all $z \in \Omega$, so $\underline{u} \in \operatorname{int} C_{+}$is a lower solution for problem (1.1).

Lemma 3.5. If hypotheses $H(f)_{1}, H(\eta, a)_{1}$ and $H(m)$ hold, then there exists $\xi^{*}>0$ such that, if $\|m\|_{\infty}<\xi^{*}$, then problem (1.1) has an upper solution: $\bar{u} \in \operatorname{int} C_{+}, \bar{u} \geqslant \underline{u}$.

Proof. We consider the following auxiliary Dirichlet problem:

$$
\begin{cases}-\Delta_{p} u(z)=m(z) a(z) \widetilde{u}(z)^{-\eta}+m(z) u(z)^{q-1}+f(z, u(z)) & \text { in } \Omega \\ \left.u\right|_{\partial \Omega}=0, & u>0\end{cases}
$$

where $\widetilde{u}=m(z)^{\frac{1}{\eta}} \underline{u}$. The Euler functional $\psi: W_{0}^{1, p}(\Omega) \longrightarrow \mathbb{R}$ for problem $(3.2)$ is defined by

$$
\begin{aligned}
\psi(u)= & \frac{1}{p}\|\nabla u\|_{p}^{p}-\frac{1}{q} \int_{\Omega} m\left(u^{+}\right)^{q} \mathrm{~d} z-\int_{\Omega} F(z, u) \mathrm{d} z-\int_{\Omega} a \underline{u}^{-\eta} u \mathrm{~d} z \\
& \forall u \in W_{0}^{1, p}(\Omega) .
\end{aligned}
$$

Clearly $\psi \in C^{1}\left(W_{0}^{1, p}(\Omega)\right)$.

Claim 1. $\psi$ satisfies the $C$-condition.

Let $\left\{u_{n}\right\}_{n \geqslant 1} \subseteq W_{0}^{1, p}(\Omega)$ be a sequence, such that

$$
\left|\psi\left(u_{n}\right)\right| \leqslant M_{1} \quad \forall n \geqslant 1
$$

for some $M_{1}>0$ and

$$
\left(1+\left\|u_{n}\right\|\right) \psi^{\prime}\left(u_{n}\right) \longrightarrow 0
$$

So, we have

$$
\left|\left\langle\psi^{\prime}\left(u_{n}\right), h\right\rangle\right| \leqslant \frac{\varepsilon_{n}\|h\|}{1+\left\|u_{n}\right\|} \quad \forall h \in W_{0}^{1, p}(\Omega),
$$


with $\varepsilon_{n} \searrow 0$. Choosing $h=-u_{n}^{-} \in W_{0}^{1, p}(\Omega)$, we see that

$$
u_{n}^{-} \longrightarrow 0 \quad \text { in } W_{0}^{1, p}(\Omega) \text {. }
$$

Next choose $h=u_{n}^{+} \in W_{0}^{1, p}(\Omega)$. Then

$$
\left\|\nabla u_{n}^{+}\right\|-\int_{\Omega} m\left(u_{n}^{+}\right)^{q} \mathrm{~d} z-\int_{\Omega} f\left(z, u_{n}^{+}\right) u_{n}^{+} \mathrm{d} z-\int_{\Omega} a \underline{u}^{-\eta} u_{n}^{+} \mathrm{d} z \leqslant \varepsilon_{n} \quad \forall n \geqslant 1 .
$$

Also, form (3.3), we have

$$
\begin{gathered}
-\left\|\nabla u_{n}^{+}\right\|+\frac{p}{q} \int_{\Omega} m\left(u_{n}^{+}\right)^{q} \mathrm{~d} z+\int_{\Omega} p F\left(z, u_{n}^{+}\right) \mathrm{d} z \\
+\int_{\Omega} p a \underline{u}^{-\eta} u_{n}^{+} \mathrm{d} z \leqslant M_{2} \quad \forall n \geqslant 1,
\end{gathered}
$$

for some $M_{2}>0$. Adding (3.5) and (3.6), we obtain

$$
\begin{aligned}
& \left(\frac{p}{q}-1\right) \int_{\Omega} m\left(u_{n}^{+}\right)^{q} \mathrm{~d} z+(p-1) \int_{\Omega} a \underline{u}^{-\eta} u_{n}^{+} \mathrm{d} z+\int_{\Omega}\left(p F\left(z, u_{n}^{+}\right)-f\left(z, u_{n}^{+}\right) u_{n}^{+}\right) \mathrm{d} z \\
& \quad \leqslant M_{2}+\varepsilon_{n} \leqslant M_{3} \quad \forall n \geqslant 1,
\end{aligned}
$$

for some $M_{3}>0$. Hypotheses $H(f)_{1}$ (iii) and (iv) imply that

$$
\beta\left(\zeta^{+}\right)^{\tau}-M_{4} \leqslant p F(z, \zeta)-f(z, \zeta) \zeta \text { for almost all } z \in \Omega \text { all } \zeta \in \mathbb{R},
$$

for some $\beta, M_{4}>0$. Using this estimate in (3.7), we obtain that the sequence $\left\{u_{n}^{+}\right\}_{n \geqslant 1} \subseteq L^{\tau}(\Omega)$ is bounded. If $p<N$ or $N<p$, let $r=p^{*}$ and if $p=N$, let $r>p$. Then we can find $t \in[0,1)$, such that

$$
\frac{1}{p}=\frac{1-t}{\tau}+\frac{t}{r}
$$

Using the interpolation inequality and the Sobolev embedding theorem (see, e.g., Gasiński and Papageorgiou [15]), we obtain

$$
\left\|\nabla u_{n}^{+}\right\|_{p}^{p} \leqslant \widehat{c}\left(1+\left\|\nabla u_{n}^{+}\right\|_{p}^{t p}+\left\|\nabla u_{n}^{+}\right\|_{p}^{q}+\left\|\nabla u_{n}^{+}\right\|_{p}\right) \quad \forall n \geqslant 1,
$$

for some $\widehat{c}>0$, so the sequence $\left\{u_{n}^{+}\right\}_{n \geqslant 1} \subseteq W_{0}^{1, p}(\Omega)$ is bounded (since $t \in$ $[0,1), q<p$ and $p>1$ ) and thus the sequence $\left\{u_{n}\right\}_{n \geqslant 1} \subseteq W_{0}^{1, p}(\Omega)$ is bounded.

So, we may assume that

$$
\begin{array}{ll}
u_{n} \longrightarrow u & \text { weakly in } W_{0}^{1, p}(\Omega), \\
u_{n} \longrightarrow u & \text { in } L^{r}(\Omega)
\end{array}
$$

and so from (3.4), we have

$$
\lim _{n \rightarrow+\infty}\left\langle A\left(u_{n}\right), u_{n}-u\right\rangle=0
$$

and using Proposition 2.2, we infer that

$$
u_{n} \longrightarrow u \text { in } W_{0}^{1, p}(\Omega) .
$$

This proves Claim 1. 
Claim 2. There exists $\xi^{*}>0$, such that, if $\|m\|_{\infty}<\xi^{*}$, then we can find $\varrho=\varrho\left(\|m\|_{\infty}\right)>0$, such that

$$
\inf _{\partial B_{\varrho}} \psi=\eta_{+}>0
$$

Hypotheses $H(f)_{1}(\mathrm{iii})$ and (v) imply that for a given $\varepsilon>0$, we can find $M_{5}=M_{5}(\varepsilon)>0$, such that

$$
F(z, \zeta) \leqslant \frac{1}{p}(\vartheta(z)+\varepsilon)\left(\zeta^{+}\right)^{p}+M_{5}\left(\zeta^{+}\right)^{r} \quad \text { for almost all } z \in \Omega \text { all } \zeta \in \mathbb{R}
$$

with $r>p$ (recall that $f(z, \zeta)=F(z, \zeta)=0$ for $\zeta \leqslant 0$ ). Hence, for every $u \in W_{0}^{1, p}(\Omega)$, we have

$$
\begin{aligned}
\psi(u) & \geqslant \frac{1}{p}\|\nabla u\|_{p}^{p}-\frac{\|m\|_{\infty}}{q}\|u\|_{q}^{q}-\frac{1}{p} \int_{\Omega} \vartheta|u|^{p} \mathrm{~d} z-\frac{\varepsilon}{p}\|u\|_{p}^{p}-M_{6}\left(\|u\|+\|u\|^{r}\right) \\
& \geqslant \frac{\xi_{0}-\varepsilon}{p}\|u\|^{p}-\frac{M_{7}\|m\|_{\infty}}{q}\|u\|^{q}-M_{7}\left(\|m\|_{\infty}\|u\|+\|u\|^{r}\right),
\end{aligned}
$$

for some $\xi_{0}, M_{6}, M_{7}>0$ (cf. e.g., Gasiński and Papageorgiou [16, p. 87, Lemma 3.1]). Choosing $\varepsilon \in\left(0, \xi_{0}\right)$, we have

$$
\psi(u) \geqslant\left(M_{8}-M_{9}\left(\frac{\|m\|_{\infty}}{q}\|u\|^{q-p}+\|m\|_{\infty}\|u\|^{1-p}+\|u\|^{r-p}\right)\right)\|u\|^{p},
$$

for some $M_{8}, M_{9}>0$. Let

$$
\mu(t)=\frac{\|m\|_{\infty}}{q} t^{q-p}+\|m\|_{\infty} t^{1-p}+t^{r-p} \quad \forall t>0 .
$$

Evidently, $\mu$ is continuous on $(0,+\infty)$ and

$$
\lim _{t \rightarrow 0^{+}} \mu(t)=\lim _{t \rightarrow+\infty} \mu(t)=+\infty .
$$

So, we can find $t_{0} \in(0,+\infty)$, such that

$$
0<\mu\left(t_{0}\right)=\inf \{\mu(t): t \geqslant 0\},
$$

hence,

$$
\mu^{\prime}\left(t_{0}\right)=0 \quad \text { and } \quad t_{0}=t_{0}\left(\|m\|_{\infty}\right)
$$

We have

$$
\frac{\|m\|_{\infty}}{q}(p-q) t_{0}^{q-p-1}+\|m\|_{\infty}(p-1) t_{0}^{-p}=(r-p) t_{0}^{r-p-1},
$$

SO

$$
\frac{\|m\|_{\infty}}{q}(p-q) t_{0}^{q-1}+\|m\|_{\infty}(p-1)=(r-p) t_{0}^{r-1} .
$$

If $t_{0} \in(0,1)$, then from $(3.9)$, we have

$$
\frac{\|m\|_{\infty}}{q}(p-q)+\|m\|_{\infty}(p-1) \geqslant(r-p) t_{0}^{r-1},
$$


so

$$
\left[\frac{\|m\|_{\infty}\left(\frac{p}{q}+p-2\right)}{r-p}\right]^{\frac{1}{r-1}} \geqslant t_{0}\left(\|m\|_{\infty}\right)
$$

and finally,

$$
t_{0}\left(\|m\|_{\infty}\right) \longrightarrow 0 \quad \text { as }\|m\|_{\infty} \searrow 0
$$

If $t_{0}>1$, then from (3.9), we have

$$
\frac{\|m\|_{\infty}}{q}(p-q) t_{0}^{q-1}+\|m\|_{\infty}(p-1) t_{0}^{q-1} \geqslant(r-p) t_{0}^{r-1},
$$

so

$$
\left[\frac{\|m\|_{\infty}\left(\frac{p}{q}+p-2\right)}{r-p}\right]^{\frac{1}{r-q}} \geqslant t_{0}\left(\left\|m_{0}\right\|_{\infty}\right)
$$

and finally,

$$
t_{0}\left(\left\|m_{0}\right\|_{\infty}\right) \longrightarrow 0 \text { as }\|m\|_{\infty} \searrow 0 .
$$

If $t_{0}=1$, then from (3.9), we have

$$
\frac{\|m\|_{\infty}}{q}(p-q)+\|m\|_{\infty}(p-1) \geqslant r-p
$$

and for $\|m\|_{\infty}$ small enough this cannot happen.

If we consider $\mu\left(t_{0}\left(\|m\|_{\infty}\right)\right)$, then

$$
\mu\left(t_{0}\right) \longrightarrow 0 \text { as }\|m\|_{\infty} \searrow 0 .
$$

and so we can find $\xi^{*} \leqslant 1$, such that

$$
\|m\|_{\infty}<\xi^{*} \quad \Longrightarrow \quad \mu\left(t_{0}\right)<\frac{M_{8}}{M_{9}},
$$

so there exists $\varrho=\varrho\left(\|m\|_{\infty}\right)=t_{0}$, such that

$$
\inf _{\partial B_{\varrho}} \psi=\eta_{+}>0 \text {. }
$$

This proves claim 2.

Claim 3. $\psi\left(t \widehat{u}_{1}\right) \longrightarrow-\infty$ as $t \rightarrow+\infty$.

Without any loss of generality, we can assume that $\tau<p$. By virtue of hypothesis $H(f)_{1}(i v)$, we can find $M_{10}>0$, such that

$$
\begin{aligned}
\frac{\mathrm{d}}{\mathrm{d} \zeta} \frac{F(z, \zeta)}{\zeta^{p}} & =\frac{f(z, \zeta) \zeta-p F(z, \zeta)}{\zeta^{p+1}} \\
& \leqslant-\beta_{0} \zeta^{\tau-p-1} \quad \text { for almost all } z \in \Omega \text { all } \zeta \geqslant M_{10} .
\end{aligned}
$$

Integrating over $[\xi, \zeta], \zeta \geqslant \xi \geqslant M_{10}$ and letting $\zeta \rightarrow+\infty$, we obtain

$$
\frac{\lambda_{1}}{p} \xi^{p}-F(z, \xi) \leqslant-\frac{\beta_{0}}{p-\tau} \xi^{\tau} \quad \text { for almost all } z \in \Omega \text { all } \xi \geqslant M_{10},
$$

So

$$
\frac{\lambda_{1}}{p} \xi^{p}-F(z, \xi) \leqslant-\frac{\beta_{0}}{p-\tau} \xi^{\tau}+M_{11} \quad \text { for almost all } z \in \Omega \text { all } \xi \geqslant 0
$$


for some $M_{11}>0$. Using this estimate, we have

$$
\psi\left(t \widehat{u}_{1}\right) \leqslant-\frac{t^{q}}{q} \int_{\Omega} m \widehat{u}_{1}^{q} \mathrm{~d} z-\frac{\beta_{0} t^{\tau}}{p-\tau}\left\|\widehat{u}_{1}\right\|_{\tau}^{\tau}+M_{12},
$$

for some $M_{12}>0$, so

$$
\psi\left(t \widehat{u}_{1}\right) \longrightarrow-\infty \text { as } t \rightarrow+\infty
$$

and this proves Claim 3 .

Claims 1, 2 and 3 permit the use of the mountain pass theorem (see Theorem 2.1) which gives $\bar{u} \in W_{0}^{1, p}(\Omega), \bar{u} \neq 0$, a critical point of $\psi$. Acting on (3.2) with $-\bar{u}^{-} \in W_{0}^{1, p}(\Omega)$, we obtain that $\bar{u} \geqslant 0, \bar{u} \neq 0$ and from nonlinear regularity theory (see e.g., Gasiński and Papageorgiou [15, pp. 737-738]) and the Vázquez maximum principle, we have that $\bar{u} \in \operatorname{int} C_{+}$.

Using the definition of $\widetilde{u}$ and the fact that $\xi^{*} \leqslant 1$, we have

$$
\begin{aligned}
-\Delta_{p} \bar{u} & \geqslant m(z) a(z) \widetilde{u}(z)^{-\eta}=a(z) \underline{u}(z)^{-\eta} \\
& \geqslant m(z) a(z) \underline{u}(z)^{-\eta} \geqslant m(z) a(z) \geqslant-\Delta_{p} \underline{u}(z),
\end{aligned}
$$

so $\bar{u} \geqslant \underline{u}$. Then

$$
\begin{aligned}
-\Delta_{p} \bar{u} & =m(z) a(z) \widetilde{u}(z)^{-\eta}+m(z) \bar{u}(z)^{q-1}+f(z, \bar{u}(z)) \\
& =a(z) \underline{u}(z)^{-\eta}+m(z) \bar{u}(z)^{q-1}+f(z, \bar{u}(z)) \\
& \geqslant a(z) \bar{u}(z)^{-\eta}+m(z) \bar{u}(z)^{q-1}+f(z, \bar{u}(z)),
\end{aligned}
$$

so $\bar{u} \in \operatorname{int} C_{+}$is an upper solution.

Using $\underline{u}, \bar{u} \in \operatorname{int} C_{+}$obtained in Lemmata 3.4 and 3.5, truncation techniques and variational methods based on the critical point theory, we will prove the first multiplicity theorem for problem (1.1).

Theorem 3.6. If hypotheses $H(f)_{1}, H(\eta, a)_{1}$ and $H(m)$ hold, then there exists $m^{*}>0$, such that, if $\|m\|_{\infty}<m^{*}$, problem (1.1) has two positive smooth solutions:

$$
u_{0}, \widehat{u} \in \text { int } C_{+} \text {with } u_{0} \leqslant \widehat{u} .
$$

Proof. Using $\underline{u}, \bar{u} \in \operatorname{int} C_{+}$, from Lemma 3.4 and Lemma 3.5, we consider the following truncation of the reaction term in (1.1):

$$
g(z, \zeta)= \begin{cases}a(z) \underline{u}(z)^{-\eta}+m(z) \underline{u}(z)^{q-1}+f(z, \underline{u}(z)) & \text { if } \zeta \leqslant \underline{u}(z), \\ a(z) \zeta^{-\eta}+m(z) \zeta^{q-1}+f(z, \zeta) & \text { if } \underline{u}(z)<\zeta<\bar{u}(z), \\ a(z) \bar{u}(z)^{-\eta}+m(z) \bar{u}(z)^{q-1}+f(z, \bar{u}(z)) & \text { if } \bar{u}(z) \leqslant \zeta .\end{cases}
$$

Evidently $g(z, \zeta)$ is a Carathéodory function. We set

$$
G(z, \zeta)=\int_{0}^{\zeta} g(z, s) \mathrm{d} s
$$


and consider the $C^{1}$-functional $\widehat{\varphi}: W_{0}^{1, p}(\Omega) \longrightarrow \mathbb{R}$, defined by

$$
\widehat{\varphi}(u)=\frac{1}{p}\|\nabla u\|_{p}^{p}-\int_{\Omega} G(z, u(z)) \mathrm{d} z \quad \forall u \in W_{0}^{1, p}(\Omega) .
$$

From (3.10), it is clear that $\widehat{\varphi}$ is coercive. Moreover, exploiting the compactness of the embedding $W_{0}^{1, p}(\Omega) \subseteq L^{p}(\Omega)$, we can easily verify that $\widehat{\varphi}$ is sequentially weakly lower semicontinuous. Hence by the Weierstrass theorem, we can find $u_{0} \in W_{0}^{1, p}(\Omega)$, such that

$$
\widehat{\varphi}\left(u_{0}\right)=\inf \left\{\widehat{\varphi}(u): u \in W_{0}^{1, p}(\Omega)\right\},
$$

so

$$
\widehat{\varphi}^{\prime}\left(u_{0}\right)=0,
$$

and thus

$$
A\left(u_{0}\right)=N_{g}\left(u_{0}\right)
$$

where $A$ is given by $(2.1)$ and $N_{g}(u)(\cdot)=g(\cdot, u(\cdot))$ for all $u \in W_{0}^{1, p}(\Omega)$.

On (3.13) we act with $\left(u_{0}-\bar{u}\right)^{+} \in W_{0}^{1, p}(\Omega)$ and using (3.10) and Lemma 3.5 , we obtain

$$
\begin{aligned}
\left\langle A\left(u_{0}\right),\left(u_{0}-\bar{u}\right)^{+}\right\rangle & =\int_{\left\{u_{0}>\bar{u}\right\}} g\left(z, u_{0}\right)\left(u_{0}-\bar{u}\right) \mathrm{d} z \\
& =\int_{\Omega}\left(a \bar{u}^{-\eta}+m \bar{u}^{q-1}+f(z, \bar{u})\right)\left(u_{0}-\bar{u}\right)^{+} \mathrm{d} z \\
& \leqslant\left\langle A(\bar{u}),\left(u_{0}-\bar{u}\right)^{+}\right\rangle
\end{aligned}
$$

so

$$
\int_{\left\{u_{0}>\bar{u}\right\}}\left(\left\|\nabla u_{0}\right\|^{p-2} \nabla u_{0}-\|\nabla \bar{u}\|^{p-2} \nabla \bar{u}, \nabla u_{0}-\nabla \bar{u}\right)_{\mathbb{R}^{N}} \mathrm{~d} x \leqslant 0,
$$

hence

$$
\left|\left\{u_{0}>\bar{u}\right\}\right|_{N}=0, \quad \text { i.e., } u_{0} \leqslant \bar{u} \text {. }
$$

In a similar fashion, acting on $(3.13)$ with $\left(\underline{u}-u_{0}\right)^{+} \in W_{0}^{1, p}(\Omega)$ and using this time Lemma 3.4, we obtain

$$
\underline{u} \leqslant u_{0} \text {. }
$$

From (3.10), (3.14) and (3.15), it follows that

$$
A\left(u_{0}\right)=a u_{0}^{-\eta}+m u_{0}^{q-1}+N_{f}\left(u_{0}\right),
$$

with $N_{f}(u)(\cdot)=f(\cdot, u(\cdot))$ for all $u \in W_{0}^{1, p}(\Omega)$, so

$$
\left\{\begin{array}{l}
-\Delta_{p} u_{0}(z)=a(z) u_{0}(z)^{-\eta}+m(z) u_{0}(z)^{q-1}+f\left(z, u_{0}(z)\right) \quad \text { in } \Omega \\
\left.u_{0}\right|_{\partial \Omega}=0
\end{array}\right.
$$


and thus, by the nonlinear regularity theory (see e.g., Gasiński and Papageorgiou [15, pp. 737-738]), we have $u_{0} \in \operatorname{int} C_{+}$and it is a (strong) solution of (1.1).

Using $u_{0} \in \operatorname{int} C_{+}$, we introduce the following truncation of the reaction in (1.1):

$$
g_{0}(z, \zeta)= \begin{cases}a(z) u_{0}(z)^{-\eta}+m(z) u_{0}(z)^{q-1}+f\left(z, u_{0}(z)\right) & \text { if } \zeta \leqslant u_{0}(z), \\ a(z) \zeta^{-\eta}+m(z) \zeta^{q-1}+f(z, \zeta) & \text { if } u_{0}(z)<\zeta .\end{cases}
$$

Evidently $g_{0}(z, \zeta)$ is a Carathéodory function. We set

$$
G_{0}(z, \zeta)=\int_{0}^{\zeta} g_{0}(z, s) \mathrm{d} s
$$

and consider the $C^{1}$-functional $\varphi_{0}: W_{0}^{1, p}(\Omega) \longrightarrow \mathbb{R}$, defined by

$$
\varphi_{0}(u)=\frac{1}{p}\|\nabla u\|_{p}^{p}-\int_{\Omega} G_{0}(z, u(z)) \mathrm{d} z \quad \forall u \in W_{0}^{1, p}(\Omega) .
$$

Claim 1. $\varphi_{0}$ satisfies the $C$-condition.

Let $\left\{u_{n}\right\}_{n \geqslant 1} \subseteq W_{0}^{1, p}(\Omega)$ be a sequence, such that

$$
\left|\varphi_{0}\left(u_{n}\right)\right| \leqslant M_{13} \quad \forall n \geqslant 1,
$$

for some $M_{13}>0$ and

$$
\left(1+\left\|u_{n}\right\|\right) \varphi_{0}^{\prime}\left(u_{n}\right) \longrightarrow 0 \quad \text { in } W^{-1, p^{\prime}}(\Omega) .
$$

From (3.20), we have

$$
\left|\left\langle A\left(u_{n}\right), h\right\rangle-\int_{\Omega} g_{0}\left(z, u_{n}\right) h \mathrm{~d} z\right| \leqslant \frac{\varepsilon_{n}\|h\|}{1+\left\|u_{n}\right\|} \quad \forall h \in W_{0}^{1, p}(\Omega),
$$

with $\varepsilon_{n} \searrow 0$. In $(3.21)$, we choose $h=u_{n} \in W_{0}^{1, p}(\Omega)$. Then

$$
\left\|\nabla u_{n}\right\|_{p}^{p}-\int_{\Omega} g_{0}\left(z, u_{n}\right) u_{n} \mathrm{~d} z \leqslant \varepsilon_{n} \quad \forall n \geqslant 1 .
$$

On the other hand, from (3.19), we have

$$
-\left\|\nabla u_{n}\right\|_{p}^{p}+\int_{\Omega} p G_{0}\left(z, u_{n}\right) \mathrm{d} z \leqslant p M_{1} \quad \forall n \geqslant 1 .
$$

Adding (3.22) and (3.23), we obtain

$$
\int_{\Omega}\left(p G_{0}\left(z, u_{n}\right)-g_{0}\left(z, u_{n}\right) u_{n}\right) \mathrm{d} z \leqslant M_{14} \quad \forall n \geqslant 1,
$$


for some $M_{14}>0$. Note that, using (3.16), we have

$$
\int_{\left\{u_{n}<u_{0}\right\}}\left(p G_{0}\left(z, u_{n}\right)-g_{0}\left(z, u_{n}\right) u_{n}\right) \mathrm{d} z \leqslant M_{15} \quad \forall n \geqslant 1,
$$

for some $M_{15}>0$. If $\eta \neq 1$, then

$$
\begin{aligned}
& \int_{\left\{u_{n} \geqslant u_{0}\right\}}\left(p G_{0}\left(z, u_{n}\right)-g_{0}\left(z, u_{n}\right) u_{n}\right) \mathrm{d} z \\
= & \int_{\left\{u_{n} \geqslant u_{0}\right\}}\left[p a u_{0}^{1-\eta}+\frac{p a}{1-\eta}\left(u_{n}^{1-\eta}-u_{0}^{1-\eta}\right)+p m u_{0}^{q}\right. \\
& +\frac{p}{q} m\left(u_{n}^{q}-u_{0}^{q}\right)+p f\left(z, u_{0}\right) u_{0} \\
& \left.+p\left(F\left(z, u_{n}\right)-F\left(z, u_{0}\right)\right)-a u_{n}^{1-\eta}-m u_{n}^{q}-f\left(z, u_{n}\right) u_{n}\right] \mathrm{d} z \\
& \\
& \quad \int_{\left\{u_{n} \geqslant u_{0}\right\}}\left(p F\left(z, u_{n}\right)-f\left(z, u_{n}\right) u_{n}\right) \mathrm{d} z \quad \forall n \geqslant 1 \\
& \left.\quad M_{16}+\quad u_{n} \geqslant u_{0}\right\}
\end{aligned}
$$

for some $M_{16}>0$.

If $\eta=1$, then

$$
\begin{aligned}
& \int_{\left\{u_{n} \geqslant u_{0}\right\}}\left(p G_{0}\left(z, u_{n}\right)-g_{0}\left(z, u_{n}\right) u_{n}\right) \mathrm{d} z \\
& =\int_{\left\{u_{n} \geqslant u_{0}\right\}}\left[p a+p a\left(\ln u_{n}-\ln u_{0}\right)+p m u_{0}^{q}+\frac{p}{q} m\left(u_{n}^{q}-u_{0}^{q}\right)+p f\left(z, u_{0}\right) u_{0}\right. \\
& \left.\quad+p\left(F\left(z, u_{n}\right)-F\left(z, u_{0}\right)\right)-a-m u_{n}^{q}-f\left(z, u_{n}\right) u_{n}\right] \mathrm{d} z \\
& \quad \int_{\left.-M_{17}+p \int_{\left\{u_{n} \geqslant u_{0}\right\}} a\left(\ln u_{n}-\ln u_{0}\right) \mathrm{d} z+u_{0}\right\}}\left(\frac{p}{q}-1\right) m u_{n}^{q} \mathrm{~d} z \\
& \quad+\int_{\left\{u_{n} \geqslant u_{0}\right\}}\left(p F\left(z, u_{n}\right)-f\left(z, u_{n}\right) u_{n}\right) \mathrm{d} z \quad \forall n \geqslant 1
\end{aligned}
$$

for some $M_{17}>0$.

If $\eta \in(0,1)$, then $\left(\frac{p}{1-\eta}-1\right)>0$. Also

$$
\ln u_{0}(z) \leqslant \ln u_{n}(z) \quad \text { for almost all } z \in\left\{u_{n} \geqslant u_{0}\right\}
$$


and since $q \in(1, p)$, we have

$$
\int_{\Omega}\left(\frac{p}{q}-1\right) m u_{n}^{q} \mathrm{~d} z \geqslant 1 \quad \forall n \geqslant 1 .
$$

Therefore, if $\eta \in(0,1]$, then (3.26) and (3.27) become

$$
\begin{aligned}
& \int_{\left\{u_{n} \geqslant u_{0}\right\}}\left(p G_{0}\left(z, u_{n}\right)-g_{0}\left(z, u_{n}\right) u_{n}\right) \mathrm{d} z \\
& \quad \geqslant-M_{18}+\int_{\left\{u_{n} \geqslant u_{0}\right\}}\left(p F\left(z, u_{n}\right)-f\left(z, u_{n}\right) u_{n}\right) \mathrm{d} z \quad \forall n \geqslant 1,
\end{aligned}
$$

for some $M_{18}>0\left(M_{18}=M_{16}\right.$ or $\left.M_{18}=M_{17}\right)$. Returning to (3.24) and using (3.25) and (3.28), we infer that, if $\eta \in(0,1]$, then

$$
\int_{\left\{u_{n} \geqslant u_{0}\right\}}\left(p F\left(z, u_{n}\right)-f\left(z, u_{n}\right) u_{n}\right) \mathrm{d} z \leqslant M_{19} \quad \forall n \geqslant 1,
$$

for some $M_{19}>0$.

If $\eta>1$, then

$$
\begin{aligned}
\left|\int_{\left\{u_{n} \geqslant u_{0}\right\}} a\left(\frac{p}{1-\eta}-1\right) u_{n}^{1-\eta} \mathrm{d} z\right| & \leqslant c_{1} \int_{\left\{u_{n} \geqslant u_{0}\right\}} a u_{n}^{1-\eta} \mathrm{d} z \\
& \leqslant c_{1} \int_{\left\{u_{n} \geqslant u_{0}\right\}} a u_{0} u_{0}^{-\eta} \mathrm{d} z \quad \forall n \geqslant 1,
\end{aligned}
$$

with $c_{1}=\left|\frac{p}{1-\eta}-1\right|>0$.

Since $u_{0} \in \operatorname{int} C_{+}$, we can find $\vartheta \in(0,1)$ small enough, such that $\vartheta \widehat{v} \leqslant u_{0}$ $\left(\widehat{v} \in C_{+}\right.$as in hypothesis $\left.H(\eta, a)_{1}\right)$. Hence from (3.30), we have

$$
\begin{aligned}
\int_{\left\{u_{n} \geqslant u_{0}\right\}} a\left(\frac{p}{1-\eta}-1\right) u_{n}^{1-\eta} \mathrm{d} z & \leqslant c_{2} \int_{\left\{u_{n} \geqslant u_{0}\right\}} \vartheta^{-\eta} a \widehat{v}^{-\eta} \mathrm{d} z \\
& \leqslant c_{2} \vartheta^{-\eta}\left\|a \widehat{v}^{-\eta}\right\|_{1} . \quad \forall n \geqslant 1,
\end{aligned}
$$

with $c_{2}=c_{1}\left\|u_{0}\right\|_{\infty}$. Returning to (3.24) and using (3.25), (3.26) and (3.31), we infer that, if $\eta>1$, then

$$
\int_{\left\{u_{n} \geqslant u_{0}\right\}}\left(p F\left(z, u_{n}\right)-f\left(z, u_{n}\right) u_{n}\right) \mathrm{d} z \leqslant M_{20} \quad \forall n \geqslant 1,
$$

for some $M_{20}>0$.

From (3.29) and (3.32), it follows that for all $\eta>0$, we have

$$
\int_{\left\{u_{n} \geqslant u_{0}\right\}}\left(p F\left(z, u_{n}\right)-f\left(z, u_{n}\right) u_{n}\right) \mathrm{d} z \leqslant M_{21} \quad \forall n \geqslant 1,
$$


for some $M_{21}>0\left(M_{21}=M_{19}\right.$ or $\left.M_{21}=M_{20}\right)$. By virtue of hypothesis $H(f)_{1}(i v)$, we can find $\beta_{1} \in\left(0, \beta_{0}\right)$ and $M_{22}>0$, such that

$$
\beta_{1} \zeta^{\tau} \leqslant p F(z, \zeta)-f(z, \zeta) \zeta \text { for almost all } z \in \Omega \text {, all } \zeta \geqslant M_{22}
$$

Moreover, hypothesis $H(f)_{1}($ iii $)$ implies that

$$
|p F(z, \zeta)-f(z, \zeta) \zeta| \leqslant M_{23} \text { for almost all } z \in \Omega \text {, all } \zeta<M_{22},
$$

for some $M_{23}>0$ (recall that $F(z, \zeta)=f(z, \zeta)=0$ for almost all $z \in \Omega$ and all $\zeta \leqslant 0)$. From (3.34) and (3.35), it follows that

$$
\beta_{1}\left(\zeta^{+}\right)^{\tau}-M_{24} \leqslant p F(z, \zeta)-f(z, \zeta) \zeta \text { for almost all } z \in \Omega \text {, all } \zeta \in \mathbb{R},
$$

for some $M_{24}>0$. Returning to (3.33) and using (3.36), we obtain

$$
\int_{\left\{u_{n} \geqslant u_{0}\right\}}\left(u_{n}^{+}\right)^{\tau} \mathrm{d} z \leqslant M_{25} \quad \forall n \geqslant 1,
$$

for some $M_{25}>0$, so

the sequence $\left\{u_{n}^{+}\right\}_{n \geqslant 1} \subseteq L^{\tau}(\Omega)$ is bounded.

On the other hand, if in (3.21) we choose $h=-u_{n}^{-} \in W_{0}^{1, p}(\Omega)$, then

$$
\left\|\nabla u_{n}^{-}\right\|_{p}^{p} \leqslant M_{26} \quad \forall n \geqslant 1,
$$

for some $M_{26}>0($ see $(3.16))$ and so the sequence $\left\{u_{n}^{-}\right\}_{n \geqslant 1} \subseteq W_{0}^{1, p}(\Omega)$ is bounded; thus finally

$$
\text { the sequence }\left\{u_{n}^{-}\right\}_{n \geqslant 1} \subseteq L^{\tau}(\Omega) \text { is bounded }
$$

(recall that $\tau \in[1, p]$; see hypothesis $H(f)_{1}(\mathrm{iv})$ ).

From (3.37) and (3.38), it follows that

$$
\text { the sequence }\left\{u_{n}\right\}_{n \geqslant 1} \subseteq L^{\tau}(\Omega) \text { is bounded }
$$

For $p<N$ or $p>N$, let $r=p^{*}$ and for $p=N$, let $r>p$. We can find $t \in[0,1)$, such that

$$
\frac{1}{p}=\frac{1-t}{\tau}+\frac{t}{r} .
$$

Invoking the interpolation inequality (see e.g., [15, Theorem A.2.28, p. 905]) and the Sobolev embedding theorem, we have

$$
\left\|u_{n}\right\|_{p} \leqslant\left\|u_{n}\right\|_{\tau}^{1-t}\left\|u_{n}\right\|_{r}^{t} \quad \forall n \geqslant 1
$$

So

$$
\left\|u_{n}\right\|_{p}^{p} \leqslant M_{27}\left\|u_{n}\right\|^{t p} \quad \forall n \geqslant 1,
$$

for some $M_{27}>0$. From hypothesis $H(f)_{1}($ iii $)$, we have

$$
\left|f\left(z, u_{n}(z)\right) u_{n}(z)\right| \leqslant c_{3}\left(1+\left|u_{n}(z)\right|^{p}\right) \quad \text { for almost all } z \in \Omega \text {, all } n \geqslant 1,
$$


for some $c_{3}>0$. Returning to (3.22) and using (3.41), (3.42) and (3.16), we obtain

$$
\left\|\nabla u_{n}\right\|_{p}^{p} \leqslant c_{4}\left(1+\left\|\nabla u_{n}\right\|_{p}^{t p}\right) \quad \forall n \geqslant 1,
$$

for some $c_{4}>0$ and so the sequence $\left\{u_{n}\right\}_{n \geqslant 1} \subseteq W_{0}^{1, p}(\Omega)$ is bounded (recall that $t \in[0,1))$.

Therefore, we may assume that

$$
\begin{array}{ll}
u_{n} \longrightarrow u & \text { weakly in } W_{0}^{1, p}(\Omega), \\
u_{n} \longrightarrow u & \text { in } L^{p}(\Omega) .
\end{array}
$$

If in (3.21) we choose $h=u_{n}-u \in W_{0}^{1, p}(\Omega)$, then

$$
\left|\left\langle A\left(u_{n}\right), u_{n}-u\right\rangle-\int_{\Omega} g_{0}\left(z, u_{n}\right)\left(u_{n}-u\right) \mathrm{d} z\right| \leqslant \frac{\varepsilon_{n}\left\|u_{n}-u\right\|}{1+\left\|u_{n}\right\|} \quad \forall n \geqslant 1,
$$

SO

$$
\lim _{n \rightarrow+\infty}\left\langle A\left(u_{n}\right), u_{n}-u\right\rangle=0
$$

and using also Proposition 2.2, we have

$$
u_{n} \longrightarrow u \text { in } W_{0}^{1, p}(\Omega),
$$

so finally $\varphi_{0}$ satisfies the $C$-condition and this proves Claim 1 .

Claim 2. $\varphi_{0}\left(t \widehat{u}_{1}\right) \longrightarrow-\infty$ as $t \rightarrow+\infty$.

Clearly in hypothesis $H(f)_{1}(\mathrm{iv})$ without any loss of generality, we may assume that $\tau<p$. We have

$$
\begin{aligned}
\frac{\mathrm{d}}{\mathrm{d} \zeta} \frac{F(z, \zeta)}{\zeta^{p}} & =\frac{f(z, \zeta) \zeta-p F(z, \zeta)}{\zeta^{p+1}} \\
& \leqslant-\beta_{1} \zeta^{\tau-p-1} \text { for almost all } z \in \Omega, \text { all } \zeta \geqslant M_{22}
\end{aligned}
$$

(see $(3.34))$, so

$$
\begin{aligned}
& \frac{F(z, \zeta)}{\zeta^{p}}-\frac{F(z, \xi)}{\xi^{p}} \\
& \quad \leqslant \frac{\beta_{1}}{p-\tau}\left(\frac{1}{\zeta^{p-\tau}}-\frac{1}{\xi^{p-\tau}}\right) \text { for almost all } z \in \Omega \text {, all } \zeta \geqslant \xi \geqslant M_{22} .
\end{aligned}
$$

Let $\zeta \rightarrow+\infty$. Recalling that $\tau<p$ and using hypothesis $H(f)_{1}(\mathrm{iv})$, we obtain

$$
\frac{\lambda_{1}}{p} \xi^{p}-F(z, \xi) \leqslant-\frac{\beta_{1}}{p-\tau} \xi^{\tau} \quad \text { for almost all } z \in \Omega \text {, all } \xi \geqslant M_{22},
$$

so

$$
\frac{\lambda_{1}}{p} \xi^{p}-F(z, \xi) \leqslant-\frac{\beta_{1}}{p-\tau} \xi^{\tau}+c_{5} \quad \text { for almost all } z \in \Omega, \text { all } \xi \geqslant 0
$$


for some $c_{5}>0$. Then, using hypothesis $H(f)_{1}(v)$, the fact that $\left\|\nabla \widehat{u}_{1}\right\|_{p}^{p}=$ $\lambda_{1}\left\|\widehat{u}_{1}\right\|_{p}^{p}$ and (3.16), we have

$$
\begin{aligned}
\varphi_{0}\left(t \widehat{u}_{1}\right) \leqslant & \frac{t^{p}}{p} \lambda_{1}\left\|\widehat{u}_{1}\right\|_{p}^{p}-\int_{\left\{t \widehat{u}_{1} \geqslant u_{0}\right\}} G_{0}\left(z, t \widehat{u}_{1}\right) \mathrm{d} z \\
\leqslant & \frac{t^{p}}{p} \lambda_{1} \int_{\left\{t \widehat{u}_{1}<u_{0}\right\}} \widehat{u}_{1}^{p} \mathrm{~d} z+\frac{t^{p}}{p} \lambda_{1} \int_{\left\{t \widehat{u}_{1} \geqslant u_{0}\right\}} \widehat{u}_{1}^{p} \mathrm{~d} z-\int_{\left\{t \widehat{u}_{1} \geqslant u_{0}\right\}} F\left(z, t \widehat{u}_{1}\right) \mathrm{d} z \\
\leqslant & \int_{\left\{t \widehat{u}_{1} \geqslant u_{0}\right\}}\left[\frac{\lambda_{1}}{p}\left(t \widehat{u}_{1}\right)^{p}-F\left(t, t \widehat{u}_{1}\right)\right] \mathrm{d} z+c_{6} \\
\leqslant & -\frac{\beta_{1} t^{\tau}}{p-\tau} \int_{\left\{t \widehat{u}_{1} \geqslant u_{0}\right\}} \widehat{u}_{1}^{\tau} \mathrm{d} z+c_{7},
\end{aligned}
$$

for some $c_{6}, c_{7}>0$. Since

$$
\chi_{\left\{t \hat{u}_{1} \geqslant u_{0}\right\}}(z) \longrightarrow \chi_{\Omega}(z) \equiv 1 \text { for almost all } z \in \Omega \text { as } t \rightarrow+\infty,
$$

from (3.45), we infer that

$$
\varphi_{0}\left(t \widehat{u}_{1}\right) \longrightarrow-\infty \quad \text { as } t \rightarrow+\infty .
$$

This proves Claim 2.

We consider the following auxiliary Dirichlet problem:

$$
\left\{\begin{array}{l}
-\Delta_{p} u(z)=g_{0}(z, u(z)) \quad \text { in } \Omega, \\
\left.u\right|_{\partial \Omega}=0 .
\end{array}\right.
$$

Note that $u_{0} \in \operatorname{int} C_{+}$is a solution of (3.46) (see (3.16)). Moreover, since $u_{0} \leqslant \underline{u}$, from (3.16), we see that $\bar{u} \in \operatorname{int} C_{+}$is still an upper solution for problem (3.46). We consider the order interval

$$
\left[u_{0}, \bar{u}\right]=\left\{u \in W_{0}^{1, p}(\Omega): u_{0}(z) \leqslant u(z) \leqslant \bar{u}(z) \text { for almost all } z \in \Omega\right\} .
$$

Note that $\varphi_{0}$ is coercive and sequentially weakly lower semicontinuous. So, we can find $\widetilde{u} \in\left[u_{0}, \bar{u}\right]$, such that

$$
\varphi_{0}(\widetilde{u})=\inf \left\{\varphi_{0}(u): u \in\left[u_{0}, \bar{u}\right]\right\} .
$$

For $y \in\left[u_{0}, \bar{u}\right]$, let

$$
\sigma_{0}(t)=\varphi(t y+(1-t) \widetilde{u}) \quad \forall t \in[0,1] .
$$

From (3.47), we have

$$
\sigma_{0}(0) \leqslant \sigma_{0}(t) \quad \forall t \in[0,1]
$$

so

$$
0 \leqslant \sigma_{0}^{\prime}(0)
$$

and thus

$$
0 \leqslant\langle A(\widetilde{u}), y-\widetilde{u}\rangle-\int_{\Omega} g_{0}(z, \widetilde{u})(y-\widetilde{u}) \mathrm{d} z \quad \forall y \in\left[u_{0}, \bar{u}\right] .
$$


Let $h \in W_{0}^{1, p}(\Omega)$ and $\varepsilon>0$. We set

$$
y(z)= \begin{cases}u_{0}(z) & \text { if } z \in\left\{\widetilde{u}+\varepsilon h \leqslant u_{0}\right\}, \\ \widetilde{u}(z)+\varepsilon h(z) & \text { if } z \in\left\{u_{0}<\widetilde{u}+\varepsilon h<\bar{u}\right\}, \\ \bar{u} & \text { if } z \in\{\bar{u} \leqslant \widetilde{u}+\varepsilon h\} .\end{cases}
$$

Evidently $y \in\left[u_{0}, \bar{u}\right]$ and we can use it as a test function in (3.48). We obtain

$$
\begin{aligned}
& 0 \leqslant \varepsilon \int_{\Omega}\|\nabla \widetilde{u}\|^{p-2}(\nabla \widetilde{u}, \nabla h)_{\mathbb{R}^{N}} \mathrm{~d} z-\varepsilon \int_{\Omega} g_{0}(z, \widetilde{u}) h \mathrm{~d} z \\
& -\int_{\{\bar{u} \leqslant \widetilde{u}+\varepsilon h\}}\left[\|\nabla \bar{u}\|^{p-2}(\nabla \bar{u}, \nabla(\widetilde{u}+\varepsilon h-\bar{u}))_{\mathbb{R}^{N}}\right. \\
& \left.-g_{0}(z, \bar{u})(\widetilde{u}+\varepsilon h-\bar{u})\right] \mathrm{d} z \\
& +\int_{\left\{\widetilde{u}+\varepsilon h \leqslant u_{0}\right\}}\left[\left\|\nabla u_{0}\right\|^{p-2}\left(\nabla u_{0}, \nabla\left(u_{0}-\tilde{u}-\varepsilon h\right)\right)_{\mathbb{R}^{N}}\right. \\
& \left.-g_{0}\left(z, u_{0}\right)\left(u_{0}-\widetilde{u}-\varepsilon h\right)\right] \mathrm{d} z \\
& +\int_{\{\bar{u} \leqslant \widetilde{u}+\varepsilon h\}}\left(g_{0}(z, \bar{u})-g_{0}(z, \widetilde{u})\right)(\bar{u}-\widetilde{u}-\varepsilon h) \mathrm{d} z \\
& +\int_{\left\{\widetilde{u}+\varepsilon h \leqslant u_{0}\right\}}\left(g_{0}\left(z, u_{0}\right)-g_{0}(z, \widetilde{u})\right)\left(u_{0}-\widetilde{u}-\varepsilon h\right) \mathrm{d} z \\
& -\int_{\left\{\widetilde{u}+\varepsilon h \leqslant u_{0}\right\}}\left(\|\nabla \widetilde{u}\|^{p-2} \nabla \widetilde{u}-\left\|\nabla u_{0}\right\|^{p-2} \nabla u_{0}, \nabla\left(\widetilde{u}-u_{0}\right)\right)_{\mathbb{R}^{N}} \\
& -\varepsilon \int_{\left\{\widetilde{u}+\varepsilon h \leqslant u_{0}\right\}}\left(\|\nabla \widetilde{u}\|^{p-2} \nabla \widetilde{u}-\left\|\nabla u_{0}\right\|^{p-2} \nabla u_{0}, \nabla h\right)_{\mathbb{R}^{N}} \\
& +\int_{\{\bar{u} \leqslant \widetilde{u}+\varepsilon h\}}\left(\|\nabla \bar{u}\|^{p-2} \nabla \bar{u}-\|\nabla \widetilde{u}\|^{p-2} \nabla \widetilde{u}, \nabla(\widetilde{u}-\bar{u})\right)_{\mathbb{R}^{N}} \\
& +\varepsilon \int_{\{\bar{u} \leqslant \widetilde{u}+\varepsilon h\}}\left(\|\nabla \bar{u}\|^{p-2} \nabla \bar{u}-\|\nabla \widetilde{u}\|^{p-2} \nabla \widetilde{u}, \nabla h\right)_{\mathbb{R}^{N}} .
\end{aligned}
$$

Recall that $\bar{u} \in \operatorname{int} C_{+}$is an upper solution for problem (3.46). Hence

$$
\begin{aligned}
& -\int_{\{\bar{u} \leqslant \widetilde{u}+\varepsilon h\}}\left[\|\nabla \bar{u}\|^{p-2}(\nabla \bar{u}, \nabla(\widetilde{u}+\varepsilon h-\bar{u}))_{\mathbb{R}^{N}}\right. \\
& \left.-g_{0}(z, \bar{u})(\widetilde{u}+\varepsilon h-\bar{u})\right] \mathrm{d} z \leqslant 0 .
\end{aligned}
$$


Also $u_{0} \in \operatorname{int} C_{+}$solves problem (3.46). Hence

$$
\begin{aligned}
& \int_{\left\{\widetilde{u}+\varepsilon h \leqslant u_{0}\right\}}\left[\left\|\nabla u_{0}\right\|^{p-2}\left(\nabla u_{0}, \nabla\left(u_{0}-\widetilde{u}-\varepsilon h\right)\right)_{\mathbb{R}^{N}}\right. \\
& \left.\quad-g_{0}\left(z, u_{0}\right)\left(u_{0}-\widetilde{u}-\varepsilon h\right)\right] \mathrm{d} z=0 .
\end{aligned}
$$

From the monotonicity of the map $\mathbb{R}^{N} \ni \xi \longmapsto\|\xi\|^{p-2} \xi$, we have

$$
-\int_{\left\{\widetilde{u}+\varepsilon h \leqslant u_{0}\right\}}\left(\|\nabla \widetilde{u}\|^{p-2} \nabla \widetilde{u}-\left\|\nabla u_{0}\right\|^{p-2} \nabla u_{0}, \nabla\left(\widetilde{u}-u_{0}\right)\right)_{\mathbb{R}^{N}} \mathrm{~d} z \leqslant 0 .
$$

and

$$
\int_{\{\bar{u} \leqslant \widetilde{u}+\varepsilon h\}}\left(\|\nabla \bar{u}\|^{p-2} \nabla \bar{u}-\|\nabla \widetilde{u}\|^{p-2} \nabla \widetilde{u}, \nabla(\widetilde{u}-\bar{u})\right)_{\mathbb{R}^{N}} \mathrm{~d} z \leqslant 0 .
$$

Note that since $\widetilde{u} \leqslant \bar{u}$, we have

$$
h(z) \geqslant 0 \quad \text { for almost all } z \in\{\bar{u} \leqslant \widetilde{u}+\varepsilon h\} .
$$

Hence using hypothesis $H(f)_{1}$ (iii) and recalling that $\widetilde{u} \leqslant \bar{u}$, we have

$$
\begin{aligned}
& \int_{\{\bar{u} \leqslant \widetilde{u}+\varepsilon h\}}\left(g_{0}(z, \bar{u})-g_{0}(z, \widetilde{u})\right)(\bar{u}-\widetilde{u}-\varepsilon h) \mathrm{d} z \\
& \leqslant-c_{8} \int_{\{\bar{u} \leqslant \widetilde{u}+\varepsilon h\}}(\bar{u}-\widetilde{u}-\varepsilon h) \mathrm{d} z \\
& \leqslant \varepsilon c_{8} \int_{\{\widetilde{u}<\bar{u} \leqslant \widetilde{u}+\varepsilon h\}} h \mathrm{~d} z
\end{aligned}
$$

for some $c_{8}>0$. In a similar fashion, we obtain

$$
\int_{\left\{\widetilde{u}+\varepsilon h \leqslant u_{0}\right\}}\left(g_{0}\left(z, u_{0}\right)-g_{0}(z, \widetilde{u})\right)\left(u_{0}-\widetilde{u}-\varepsilon h\right) \mathrm{d} z \leqslant-\varepsilon c_{9} \int_{\left\{\widetilde{u}+\varepsilon h \leqslant u_{0}<\widetilde{u}\right\}} h \mathrm{~d} z
$$

for some $c_{9}>0$. 
We return to (3.49) and use (3.50)-(3.55). Then

$$
\begin{aligned}
0 \leqslant & \int_{\Omega}\|\nabla \widetilde{u}\|^{p-2}(\nabla \widetilde{u}, \nabla h)_{\mathbb{R}^{N}}-\int_{\Omega} g_{0}(z, \widetilde{u}) h \mathrm{~d} z \\
& +c_{8} \int_{\{\widetilde{u}<\bar{u} \leqslant \widetilde{u}+\varepsilon h\}} h \mathrm{~d} z-c_{9} \int_{\left\{\widetilde{u}+\varepsilon h \leqslant u_{0}<\widetilde{u}\right\}} h \mathrm{~d} z \\
& -\int_{\left\{\widetilde{u}+\varepsilon h \leqslant u_{0}\right\}}\left(\|\nabla \widetilde{u}\|^{p-2} \nabla \widetilde{u}-\left\|\nabla u_{0}\right\|^{p-2} \nabla u_{0}, \nabla h\right)_{\mathbb{R}^{N}} \mathrm{~d} z \\
& +\int_{\{\bar{u} \leqslant \widetilde{u}+\varepsilon h\}}\left(\|\nabla \bar{u}\|^{p-2} \nabla \bar{u}-\|\nabla \widetilde{u}\|^{p-2} \nabla \widetilde{u}, \nabla h\right)_{\mathbb{R}^{N}} \mathrm{~d} z .
\end{aligned}
$$

Stampacchia's theorem (see, e.g., Gasiński and Papageorgiou [15, p. 195]) implies that

$$
\begin{aligned}
& \nabla \widetilde{u}(z)=\nabla u_{0}(z) \quad \text { for almost all } z \in\left\{\widetilde{u}=u_{0}\right\}, \\
& \nabla \widetilde{u}(z)=\nabla \bar{u}(z) \quad \text { for almost all } z \in\{\widetilde{u}=\bar{u}\} .
\end{aligned}
$$

Therefore, if in (3.56) we let $\varepsilon \searrow 0$, then

$$
0 \leqslant \int_{\Omega}\|\nabla \widetilde{u}\|^{p-2}(\nabla \widetilde{u}, \nabla h)_{\mathbb{R}^{N}} \mathrm{~d} z-\int_{\Omega} g_{0}(z, \widetilde{u}) h \mathrm{~d} z \quad \forall h \in W_{0}^{1, p}(\Omega),
$$

So

$$
A(\widetilde{u})=N_{g_{0}}(\widetilde{u}),
$$

with $N_{g_{0}}(u)(\cdot)=g_{0}(\cdot, u(\cdot))$ for all $u \in W_{0}^{1, p}(\Omega)$.

If $\widetilde{u} \neq u_{0}$, then from (3.57) we infer that $\widetilde{u}$ solves (1.1) (see (3.16)) and $\widetilde{u} \in \operatorname{int} C_{+}$(nonlinear regularity theory; see e.g., Gasiński and Papageorgiou [15, pp. 737-738]).

If $\widetilde{u}=u_{0}$, then we may assume that $u_{0}$ is an isolated critical point of $\varphi_{0}$ (otherwise we have a whole sequence of distinct positive smooth solutions of (1.1) converging in $W_{0}^{1, p}(\Omega)$ to $u_{0}$, since the critical points $u$ of $\varphi_{0}$ satisfy $u \geqslant u_{0}$; see (3.16)). Arguing as in Motreanu et al. [25] (see the proof of Proposition 6$)$, we can find $\varrho \in(0,1)$ small enough, such that

$$
\varphi_{0}\left(u_{0}\right)<\inf \left\{\varphi_{0}(u):\left\|u-u_{0}\right\|=\varrho\right\}=\eta_{\varrho} .
$$

Because of (3.58) and Claims 1 and 2, we can apply the mountain pass theorem (see Theorem 2.1) and obtain $\widehat{u} \in W_{0}^{1, p}(\Omega)$, such that

$$
\varphi_{0}\left(u_{0}\right)<\eta_{\varrho} \leqslant \varphi_{0}(\widehat{u})
$$

$($ see $(3.58))$ and

$$
\varphi_{0}^{\prime}(\widehat{u})=0 .
$$

From (3.58), we have that $\widehat{u} \neq u_{0}$. From (3.59), we have

$$
A(\widehat{u})=N_{g_{0}}(\widehat{u}) \text { and } \widehat{u} \geqslant u_{0} .
$$


From (3.61) it follows that $\widehat{u}$ is a solution of (1.1), $\widehat{u} \in \operatorname{int} C_{+}$(nonlinear regularity theory) and $\widehat{u} \geqslant u_{0}, \widehat{u} \neq u_{0}$.

\section{4. $(p-1)$-Superlinear Perturbation}

In this section we investigate the case where $f(z, \cdot)$ is $(p-1)$-superlinear near $+\infty$. However, we do not employ the usual in such cases Ambrosetti-Rabinowitz condition. We recall that the Ambrosetti-Rabinowitz condition says that there exist $\mu>p$ and $M>0$, such that

$$
f(z, \zeta) \zeta \geqslant \mu F(z, \zeta) \text { for almost all } z \in \Omega \text {, all } \zeta \geqslant M .
$$

Here, we state the Ambrosetti-Rabinowitz condition on the positive semiaxis since in our problem $f(z, \zeta)=F(z, \zeta)=0$ for almost all $z \in \Omega$ and all $\zeta \leqslant 0$. Integrating (4.1), we obtain the weaker condition

$$
c_{10} \zeta^{\mu} \leqslant F(z, \zeta) \text { for almost all } z \in \Omega \text {, all } \zeta \geqslant M,
$$

for some $c_{10}>0$. Then (4.2) implies the much weaker condition

$$
\lim _{\zeta \rightarrow+\infty} \frac{F(z, \zeta)}{\zeta^{p}}=+\infty \quad \text { for almost all } z \in \Omega .
$$

The precise hypotheses on the nonlinearity $f$ are the following:

$\underline{H(f)_{2}} f: \Omega \times \mathbb{R} \longrightarrow \mathbb{R}$ is a function, such that

(i) for all $\zeta \in \mathbb{R}$, the function $z \longmapsto f(z, \zeta)$ is measurable;

(ii) for almost all $z \in \Omega$, the function $\zeta \longmapsto f(z, \zeta)$ is continuous and we have $f(z, 0)=0$;

(iii) for almost all $z \in \Omega$ and all $\zeta \in \mathbb{R}$, we have

$$
|f(z, \zeta)| \leqslant a(z)+c|\zeta|^{r-1},
$$

with $a \in L^{\infty}(\Omega)_{+}, c>0, p<r<p^{*}$;

(iv) if $F(z, \zeta)=\int_{0}^{\zeta} f(z, s) \mathrm{d} s$, then

$$
\lim _{\zeta \rightarrow+\infty} \frac{F(z, \zeta)}{\zeta^{p}}=+\infty,
$$

uniformly for almost all $z \in \Omega$ and there exist $\widehat{\beta}_{0}>0$ and $\tau>q, \tau \in$ $\left((r-p) \max \left\{1, \frac{N}{p}\right\}, p^{*}\right)$, such that

$$
\liminf _{\zeta \rightarrow+\infty} \frac{f(z, \zeta) \zeta-p F(z, \zeta)}{\zeta^{\tau}} \geqslant \widehat{\beta}_{0}
$$

uniformly for almost all $z \in \Omega$;

(v) we have

$$
\lim _{\zeta \rightarrow 0^{+}} \frac{f(z, \zeta)}{\zeta^{p-1}}=0
$$

uniformly for almost all $z \in \Omega$ and

$$
m(z) \zeta^{q-1}+f(z, \zeta) \geqslant 0 \text { for almost all } z \in \Omega \text { and all } \zeta \geqslant 0 \text {. }
$$


Remark 4.1. Note that the first part of hypothesis $H(f)_{2}(i v)$ implies that for almost all $z \in \Omega, F(z, \cdot)$ is $p$-superlinear near $+\infty$. This is true if for almost all $z \in \Omega, f(z, \zeta)$ is $(p-1)$-superlinear near $+\infty$. However, instead of the Ambrosetti-Rabinowitz condition (4.1), we use a weaker condition (see the second part of hypothesis $H(f)_{2}(i v)$. Similar conditions can be found in Costa and Magalhães [8] and Fei [12]).

Example 4.2. The following function $f$ satisfies hypotheses $H(f)_{2}$ (again for the sake of simplicity we drop the $z$-dependence):

$$
f(\zeta)= \begin{cases}0 & \text { if } \zeta \leqslant 0, \\ \zeta^{r-1} & \text { if } 0<\zeta<1, \\ \zeta^{p-1}\left(\ln \zeta+\frac{1}{p}\right)+\frac{p-1}{p} & \text { if } 1 \leqslant \zeta,\end{cases}
$$

with $p<r<p^{*}$ Note that this $f$ does not satisfy the Ambrosetti-Rabinowitz condition.

Hypothesis $H(\eta, a)_{1}$ is strengthened as follows:

$H(\eta, a)_{2} \eta>0, a(z) \geqslant 0$ for almost all $z \in \Omega$ and there exist $\widehat{v} \in C_{+}$and $\overline{s>N, s} \geqslant \tau^{\prime}$ (with $\frac{1}{\tau}+\frac{1}{\tau^{\prime}}=1$, where $\tau$ is as in hypothesis $H(f)_{2}(\mathrm{iv})$ ), such that $a \widehat{v}^{-\eta} \in L^{s}(\Omega)$.

Theorem 4.3. If hypotheses $H(f)_{2}, H(\eta, a)_{2}$ and $H(m)$ hold, then there exists $m^{*}>0$, such that, if $\|m\|_{\infty}<m^{*}$, problem (1.1) has two positive smooth solutions:

$$
u_{0}, \widehat{u} \in \text { int } C_{+} \quad \text { with } \quad u_{0} \leqslant \widehat{u} .
$$

Proof. A careful reading of the proofs of Lemmata 3.4 and 3.5 reveals that $\underline{u}, \bar{u} \in \operatorname{int} C_{+}$are still lower and upper solutions for problem (1.1) under the new hypotheses $H(f)_{2}$. Again, we truncate the reaction term of (1.1) at the pair $\{\underline{u}, \bar{u}\}$, produce the Carathéodory function $g(z, \zeta)$ (see $(3.10))$, set $G(z, \zeta)$ as in (3.11) and then introduce the $C^{1}$-functional $\widehat{\varphi}: W_{0}^{1, p}(\Omega) \longrightarrow \mathbb{R}$, defined by

$$
\widehat{\varphi}(u)=\frac{1}{p}\|\nabla u\|_{p}^{p}-\int_{\Omega} G(z, u(z)) \mathrm{d} z \quad \forall u \in W_{0}^{1, p}(\Omega) .
$$

(cf. (3.12)). This functional is coercive and sequentially weakly lower semicontinuous. So, we can find $u_{0} \in W_{0}^{1, p}(\Omega)$, such that

$$
\widehat{\varphi}\left(u_{0}\right)=\inf \left\{\widehat{\varphi}(u): u \in W_{0}^{1, p}(\Omega)\right\} \quad \text { and } \quad \hat{\varphi}^{\prime}\left(u_{0}\right)=0 .
$$

We obtain $u_{0} \in[\underline{u}, \bar{u}]$ and so $u_{0} \in \operatorname{int} C_{+}$solves (1.1) (see the proof of Theorem 3.6).

Then as earlier truncate the reaction term at $u_{0}$ and produce the Carathéodory function $g_{0}(z, \zeta)$ (see $\left.(3.16)\right)$. We set $G_{0}(z, \zeta)$ as in $(3.17)$ and consider the $C^{1}$-functional $\varphi_{0}: W_{0}^{1, p}(\Omega) \longrightarrow \mathbb{R}$, defined by 


$$
\varphi_{0}(u)=\frac{1}{p}\|\nabla u\|_{p}^{p}-\int_{\Omega} G_{0}(z, u(z)) \mathrm{d} z \quad \forall u \in W_{0}^{1, p}(\Omega) .
$$

(cf. (3.18)).

Claim 1. $\varphi_{0}$ satisfies the $C$-condition.

Let $\left\{u_{n}\right\}_{n \geqslant 1} \subseteq W_{0}^{1, p}(\Omega)$ be a sequence, such that

$$
\left|\varphi_{0}\left(u_{n}\right)\right| \leqslant M_{28} \quad \forall n \geqslant 1,
$$

for some $M_{28}>0$ and

$$
\left(1+\left\|u_{n}\right\|\right) \varphi_{0}^{\prime}\left(u_{n}\right) \longrightarrow 0 \quad \text { in } W^{-1, p^{\prime}}(\Omega) .
$$

From (4.4), we have

$$
\left|\left\langle A\left(u_{n}\right), h\right\rangle-\int_{\Omega} g_{0}\left(z, u_{n}\right) h \mathrm{~d} z\right| \leqslant \frac{\varepsilon_{n}\|h\|}{1+\left\|u_{n}\right\|} \quad \forall h \in W_{0}^{1, p}(\Omega),
$$

with $\varepsilon_{n} \searrow 0$. In (4.5), we choose $h=-u_{n}^{-} \in W_{0}^{1, p}(\Omega)$. Then

$$
\begin{aligned}
& \left|\left\|\nabla u_{n}^{-}\right\|_{p}^{p}-\int_{\Omega} a u_{0}^{-\eta}\left(-u_{n}^{-}\right) \mathrm{d} z-\int_{\Omega} m u_{0}^{q-1}\left(-u_{n}^{-}\right) \mathrm{d} z-\int_{\Omega} f\left(z, u_{0}\right)\left(-u_{n}^{-}\right) \mathrm{d} z\right| \\
& \quad \leqslant \varepsilon_{n} \quad \forall n \geqslant 1
\end{aligned}
$$

(see (3.16)). Thus

$$
\left\|\nabla u_{n}^{-}\right\|_{p}^{p} \leqslant c_{11}\left(1+\left\|\nabla u_{n}^{-}\right\|_{p}\right) \quad \forall n \geqslant 1
$$

for some $c_{11}>0$ and so

$$
\text { the sequence }\left\{u_{n}^{-}\right\}_{n \geqslant 1} \subseteq L^{\tau}(\Omega) \text { is bounded. }
$$

Next in (4.5), we choose $h=u_{n}^{+} \in W_{0}^{1, p}(\Omega)$. Then

$$
-\left\|\nabla u_{n}^{+}\right\|_{p}^{p}+\int_{\Omega} g_{0}\left(z, u_{n}\right) u_{n}^{+} \mathrm{d} z \leqslant \varepsilon_{n} \quad \forall n \geqslant 1 .
$$

Also, from (4.3) and (4.6), we have

$$
\left\|\nabla u_{n}^{+}\right\|_{p}^{p}-\int_{\Omega} p G_{0}\left(z, u_{n}^{+}\right) \mathrm{d} z \leqslant M_{29} \quad \forall n \geqslant 1,
$$

for some $M_{29}>0$. We add (4.7) and (4.8) and obtain

$$
\int_{\Omega}\left(g_{0}\left(z, u_{n}^{+}\right) u_{n}^{+}-p G_{0}\left(z, u_{n}^{+}\right)\right) \mathrm{d} z \leqslant M_{30} \quad \forall n \geqslant 1,
$$


for some $M_{30}>0$. We proceed similarly as in the proof of Theorem 3.6. First assume that $\eta \neq 1$. Then

$$
\begin{aligned}
& \int_{\Omega}\left(g_{0}\left(z, u_{n}^{+}\right) u_{n}^{+}-p G_{0}\left(z, u_{n}^{+}\right)\right) \mathrm{d} z \\
& \geqslant-M_{31}+\int_{\left\{u_{n} \geqslant u_{0}\right\}} a\left(1-\frac{p}{1-\eta}\right) u_{n}^{1-\eta} \mathrm{d} z+\int_{\left\{u_{n} \geqslant u_{0}\right\}}\left(1-\frac{p}{q}\right) m u_{n}^{q} \mathrm{~d} z \\
& \quad+\int_{\left\{u_{n} \geqslant u_{0}\right\}}\left(f\left(z, u_{n}\right) u_{n}-p F\left(z, u_{n}\right)\right) \mathrm{d} z \quad \forall n \geqslant 1
\end{aligned}
$$

for some $M_{31}>0$.

If $\eta>1$, then $a\left(1-\frac{p}{1-\eta}\right)>0$ and so from (4.9) and (4.10), we have

$$
\begin{aligned}
& \int_{\left\{u_{n} \geqslant u_{0}\right\}}\left(f\left(z, u_{n}\right) u_{n}-p F\left(z, u_{n}\right)\right) \mathrm{d} z \\
& \quad \leqslant M_{32}+\left(\frac{p}{q}-1\right) \int_{\left\{u_{n} \geqslant u_{0}\right\}} m u_{n}^{q} \mathrm{~d} z \quad \forall n \geqslant 1,
\end{aligned}
$$

with $M_{32}=M_{30}+M_{31}>0$.

By virtue of hypothesis $H(f)_{2}(\mathrm{iv})$, we can find $\widehat{\beta}_{1} \in\left(0, \widehat{\beta}_{0}\right)$ and $M_{33}>0$, such that

$$
\widehat{\beta}_{1} \zeta^{\tau} \leqslant f(z, \zeta) \zeta-p F(z, \zeta) \text { for almost all } z \in \Omega \text {, all } \zeta \geqslant M_{33}
$$

Combining this estimate with hypothesis $H(f)_{2}(i i i)$, we obtain

$$
\widehat{\beta}_{1} \zeta^{\tau}-c_{12} \leqslant f(z, \zeta) \zeta-p F(z, \zeta) \text { for almost all } z \in \Omega, \text { all } \zeta \in \mathbb{R},(4.12)
$$

for some $c_{12}>0$. Using (4.12) in (4.11), we obtain

$$
\widehat{\beta}_{1} \int_{\left\{u_{n} \geqslant u_{0}\right\}} u_{n}^{\tau} \mathrm{d} \tau \leqslant M_{34}+\left(\frac{p}{q}-1\right)\|m\|_{\infty} \int_{\left\{u_{n} \geqslant u_{0}\right\}} u_{n}^{q} \mathrm{~d} \tau \quad \forall n \geqslant 1,
$$

for some $M_{34}>0$. 
Now suppose that $\eta \in(0,1)$. Then, since $\vartheta \widehat{v} \leqslant u_{0}$ for some $\vartheta \in(0,1)$ and recalling that $\tau \geqslant s^{\prime}$ (see hypothesis $H(\eta, a)_{2}$ ), we have

$$
\begin{aligned}
\int_{\left\{u_{n} \geqslant u_{0}\right\}} a\left(1-\frac{p}{1-\eta}\right) u_{n}^{1-\eta} \mathrm{d} z \mid & \leqslant c_{13} \int_{\left\{u_{n} \geqslant u_{0}\right\}} a u_{n}^{1-\eta} \mathrm{d} z \\
& \leqslant c_{13} \int_{\left\{u_{n} \geqslant u_{0}\right\}} a u_{0}^{-\eta} u_{n} \mathrm{~d} z \\
& \leqslant c_{13} \int_{\left\{u_{n} \geqslant u_{0}\right\}} \vartheta^{-\eta} a \widehat{v}^{-\eta} u_{n} \mathrm{~d} z \\
& \leqslant c_{14}\left\|u_{n}^{+}\right\|_{\tau} \forall n \geqslant 1 .
\end{aligned}
$$

with $c_{13}=\left|1-\frac{p}{1-\eta}\right|>0$ and for some $c_{14}>0$. Using (4.9), (4.12) and (4.14) in $(4.10)$, we obtain

$$
\begin{aligned}
\widehat{\beta}_{1} \int_{\left\{u_{n} \geqslant u_{0}\right\}} u_{n}^{\tau} \mathrm{d} z \leqslant & M_{35}+c_{14}\left\|u_{n}^{+}\right\|_{\tau} \\
& +\left(\frac{p}{q}-1\right)\|m\|_{\infty} \int_{\left\{u_{n} \geqslant u_{0}\right\}} u_{n}^{q} \mathrm{~d} z \quad \forall n \geqslant 1,
\end{aligned}
$$

for some $M_{35}>0$ (recall that $\eta \in(0,1)$ ).

Finally let $\eta=1$. Then

$$
\begin{aligned}
& \int_{\Omega}\left(g_{0}\left(z, u_{n}^{+}\right) u_{n}^{+}-p G_{0}\left(z, u_{n}^{+}\right)\right) \mathrm{d} z \\
& \geqslant-M_{36}+p \int_{\left\{u_{n} \geqslant u_{0}\right\}} a\left(\ln u_{0}-\ln u_{n}\right) \mathrm{d} z+\int_{\left\{u_{n} \geqslant u_{0}\right\}}\left(1-\frac{p}{q}\right) m u_{n}^{q} \mathrm{~d} z \\
& \quad+\int_{\left\{u_{n} \geqslant u_{0}\right\}}\left(f\left(z, u_{n}\right) u_{n}-p F\left(z, u_{n}\right)\right) \mathrm{d} z \quad \forall n \geqslant 1,
\end{aligned}
$$

for some $M_{36}>0$.

From (4.9) and (4.16), we have

$$
\begin{gathered}
M_{37}+p \int_{\left\{u_{n} \geqslant u_{0}\right\}} a \ln u_{n} \mathrm{~d} z+\left(\frac{p}{q}-1\right)\|m\|_{\infty} \int_{\left\{u_{n} \geqslant u_{0}\right\}} u_{n}^{q} \mathrm{~d} z \\
\geqslant \int_{\left\{u_{n} \geqslant u_{0}\right\}}\left(f\left(z, u_{n}\right) u_{n}-p F\left(z, u_{n}\right)\right) \mathrm{d} z \quad \forall n \geqslant 1,
\end{gathered}
$$


for some $M_{37}>0$, so

$$
\begin{gathered}
M_{38}+p \int_{\left\{u_{n} \geqslant u_{0}\right\}} a u_{n} \mathrm{~d} z+\left(\frac{p}{q}-1\right)\|m\|_{\infty} \int_{\left\{u_{n} \geqslant u_{0}\right\}} u_{n}^{q} \mathrm{~d} z \\
\geqslant \widehat{\beta}_{1} \int_{\left\{u_{n} \geqslant u_{0}\right\}} u_{n}^{\tau} \mathrm{d} z \quad \forall n \geqslant 1,
\end{gathered}
$$

for some $M_{38}>0$ (recall that $\eta=1$ and see (4.12)).

From $(4.13),(4.15),(4.17)$ and since $q<p, \tau>q$, we infer that

$$
\text { the sequence }\left\{u_{n}^{+}\right\}_{n \geqslant 1} \subseteq L^{\tau}(\Omega) \text { is bounded. }
$$

It is clear from hypothesis $H(f)_{2}$ (iv) that we can always assume that $\tau \leqslant r<$ $p^{*}$. Then for $p<N$ or $p>N$, we can find $t \in[0,1)$, such that

$$
\frac{1}{r}=\frac{1-t}{\tau}+\frac{t}{p^{*}}
$$

Invoking the interpolation inequality, we have

$$
\left\|u_{n}^{+}\right\|_{r} \leqslant\left\|u_{n}^{+}\right\|_{\tau}^{1-t}\left\|u_{n}^{+}\right\|_{p^{*}}^{t},
$$

So

$$
\left\|u_{n}^{+}\right\|_{r}^{r} \leqslant M_{39}\left\|u_{n}^{+}\right\|_{p^{*}}^{t r} \quad \forall n \geqslant 1,
$$

for some $M_{39}>0$. From (4.5), by choosing $h=u_{n}^{+} \in W_{0}^{1, p}(\Omega)$, we have

$$
\begin{gathered}
\left\|\nabla u_{n}^{+}\right\|_{p}^{p}-\int_{\left\{u_{n} \geqslant u_{0}\right\}} a u_{n}^{1-\eta} \mathrm{d} z-\int_{\left\{u_{n} \geqslant u_{0}\right\}} m u_{n}^{q} \mathrm{~d} z \\
-\int_{\left\{u_{n} \geqslant u_{0}\right\}} f\left(z, u_{n}\right) u_{n} \mathrm{~d} z \leqslant M_{40} \quad \forall n \geqslant 1,
\end{gathered}
$$

for some $M_{40}>0$.

From hypotheses $H(f)_{2}$ (iii) and (iv), we see that for a given $\varepsilon>0$, we can find $c_{15}=c_{15}(\varepsilon)>0$, such that

$$
|f(z, \zeta) \zeta| \leqslant \varepsilon|\zeta|^{p}+c_{15}|\zeta|^{r} \quad \text { for almost all } z \in \Omega, \text { all } \zeta \in \mathbb{R} \text {. }
$$

Combining (4.20), (4.21), (4.22) and the Sobolev embedding theorem, we have

$$
\left\|\nabla u_{n}^{+}\right\|_{p}^{p} \leqslant c_{16}\left(1+\left\|u_{n}^{+}\right\|+\left\|u_{n}^{+}\right\|^{q}+\varepsilon\left\|u_{n}^{+}\right\|_{p}^{p}+\left\|u_{n}^{+}\right\|^{t r}\right) \quad \forall n \geqslant 1,
$$

for some $c_{16}>0$.

If $p=N$, then we may assume that $\tau<r$. Also let $\mu>r$. We have

$$
\frac{1}{r}=\frac{1-t}{\tau}+\frac{t}{\mu}
$$

for some $t \in(0,1)$, so

$$
t r=\frac{\mu(r-\tau)}{\mu-\tau} .
$$


Note that

$$
\frac{\mu(r-\tau)}{\mu-\tau} \longrightarrow r-\tau \quad \text { as } \mu \rightarrow+\infty=p^{*}
$$

and by hypothesis $H(f)_{2}(i v)$, we have $r-\tau<p$. Therefore, for $\mu>r$ large enough, we have $\frac{\mu(r-\tau)}{\mu-\tau}<p$ and so $t r<p$. We repeat the argument of the case $p<N$ or $N<p$, with $p^{*}$ replaced by this large $\mu>r$. Using the Sobolev embedding theorem, we reach again (4.23).

Suppose that $\left\|u_{n}^{+}\right\| \longrightarrow+\infty$ and set

$$
y_{n}=\frac{u_{n}^{+}}{\left\|u_{n}^{+}\right\|} \quad \forall n \geqslant 1
$$

Then

$$
\left\|y_{n}\right\|=1 \quad \forall n \geqslant 1
$$

and so we may assume that

$$
\begin{array}{ll}
y_{n} \longrightarrow y & \text { weakly in } W_{0}^{1, p}(\Omega), \\
y_{n} \longrightarrow y & \text { in } L^{p}(\Omega) .
\end{array}
$$

From (4.23), we have

$$
\left\|\nabla y_{n}\right\|_{p}^{p} \leqslant c_{16}\left(\frac{1}{\left\|u_{n}^{+}\right\|^{p}}+\frac{1}{\left\|u_{n}^{+}\right\|^{p-1}}+\frac{1}{\left\|u_{n}^{+}\right\|^{p-q}}+\varepsilon\left\|y_{n}\right\|_{p}^{p}+\frac{1}{\left\|u_{n}^{+}\right\|^{p-t r}}\right) .
$$

The hypothesis on $\tau$ (see hypothesis $\left.H(f)_{2}(\mathrm{iv})\right)$ implies that $t r<p$. So, if we pass to the limit as $n \rightarrow+\infty$, then

$$
\|\nabla y\|_{p}^{p} \leqslant \varepsilon c_{16}\|y\|_{p}^{p} \leqslant \frac{\varepsilon}{\lambda_{1}} c_{16}
$$

(see (4.24) and note that $\|y\|_{p}^{p} \leqslant \frac{1}{\lambda_{1}}\|\nabla y\|_{p}^{p}=\frac{1}{\lambda_{1}}$; see $(2.3)$ ).

Since $\varepsilon>0$ is arbitrary, we let $\varepsilon \searrow 0$ and obtain $y=0$. Then

$$
\nabla y_{n} \longrightarrow 0 \quad \text { in } L^{p}\left(\Omega ; \mathbb{R}^{N}\right)
$$

so

$$
y_{n} \longrightarrow 0 \quad \text { in } W_{0}^{1, p}(\Omega),
$$

a contradiction to the fact that $\left\|y_{n}\right\|=1$ for all $n \geqslant 1$. This proves that the sequence $\left\{u_{n}^{+}\right\}_{n \geqslant 1} \subseteq W_{0}^{1, p}(\Omega)$ is bounded, which combined with (4.6) implies that the sequence $\left\{u_{n}\right\}_{n \geqslant 1} \subseteq W_{0}^{1, p}(\Omega)$ is bounded. So, we may assume that

$$
\begin{aligned}
& u_{n} \longrightarrow u \text { weakly in } W_{0}^{1, p}(\Omega), \\
& u_{n} \longrightarrow u \text { in } L^{r}(\Omega) .
\end{aligned}
$$

If in (4.5) we choose $h=u_{n}-u \in W_{0}^{1, p}(\Omega)$, pass to the limit as $n \rightarrow+\infty$ and use (4.26), then

$$
\lim _{n \rightarrow+\infty}\left\langle A\left(u_{n}\right), u_{n}-u\right\rangle=0
$$


so from (4.26) and Proposition 2.2, we have

$$
u_{n} \longrightarrow u \quad \text { in } W_{0}^{1, p}(\Omega)
$$

and so $\varphi_{0}$ satisfies the $C$-condition.

This proves Claim 1.

Claim 2. $\varphi_{0}\left(t \widehat{u}_{1}\right) \longrightarrow-\infty$ as $t \rightarrow+\infty$.

By virtue of the first part of hypothesis $H(f)_{2}(\mathrm{iv})$, for a given $\xi>0$, we can find $M_{41}=M_{41}(\xi)>0$, such that

$$
F(z, \zeta) \geqslant \frac{\xi}{p} \zeta^{p} \quad \text { for almost all } z \in \Omega, \text { all } \zeta \geqslant M_{41}
$$

Since $\widehat{u}_{1} \in \operatorname{int} C_{+}$, for $t>0$ large enough, we have $t \widehat{u}_{1} \geqslant u_{0}$. Hence for such large $t>0$, we have

$$
\varphi_{0}\left(t \widehat{u}_{1}\right) \leqslant \frac{t^{p}}{p} \lambda_{1}-\int_{\Omega} F\left(z, t \widehat{u}_{1}\right) \mathrm{d} z+c_{17} \leqslant \frac{t^{p}}{p} \lambda_{1}-\frac{t^{p}}{p} \xi+c_{17},
$$

for some $c_{17}>0$ (see $(3.16)$ and (4.28)). So

$$
\varphi_{0}\left(t \widehat{u}_{1}\right) \leqslant \frac{t^{p}}{p}\left(\lambda_{1}-\xi\right)+c_{17} .
$$

Choose $\xi>\lambda_{1}$. Then from (4.29), it is clear that

$$
\varphi_{0}\left(t \widehat{u}_{1}\right) \longrightarrow-\infty \text { as } t \rightarrow+\infty \text {. }
$$

As in the proof of Theorem 3.6, we consider the auxiliary Dirichlet problem (3.46). Recall that $u_{0} \in \operatorname{int} C_{+}$is a solution of (3.46) (see (3.16)) and $\bar{u} \in \operatorname{int} C_{+}$ is an upper solution of (3.46). We proceed as in the proof of Theorem 3.6 and using Claims 1, 2 and the mountain pass theorem (see Theorem 3.6), we obtain $\widehat{u} \in \operatorname{int} C_{+}, u_{0} \leqslant \widehat{u}, u_{0} \neq \widehat{u}$, a second positive solution of (1.1).

\section{Acknowledgements}

The authors wish to thank a knowledgeable referee for bringing to their attention additional important works on the subject.

Open Access. This article is distributed under the terms of the Creative Commons Attribution Noncommercial License which permits any noncommercial use, distribution, and reproduction in any medium, provided the original author(s) and source are credited.

\section{References}

[1] Agarwal, R.P., Lü, H., O'Regan, D.: Existence theorems for the one-dimensional singular $p$-Laplacian equation with sign changing nonlinearities. Appl. Math. Comput. 143, 15-38 (2003)

[2] Agarwal, R.P., O'Regan, D.: Singular initial and boundary value problems with sign changing nonlinearities. IMA J. Appl. Math. 65, 173-198 (2000) 
[3] Agarwal, R.P., Perera, K., O'Regan, D.: A variational approach in singular quasilinear elliptic problems with sign changing nonlinearities. Appl. Anal. 85, 12011206 (2006)

[4] Ambrosetti, A., Brézis, H., Cerami, G.: Combined effects of concave and convex nonlinearities in some elliptic problems. J. Funct. Anal. 122, 519-543 (1994)

[5] Bartolo, P., Benci, V., Fortunato, D.: Abstract critical point theorems and applications to some nonlinear problems with "strong" resonance at infinity. Nonlinear Anal. 7, 981-1012 (1983)

[6] Brock, F., Itturiaga, L., Ubilla, P.: A multiplicity result for the $p$-Laplacian involving a parameter. Ann. Henri Poincaré 9, 1371-1386 (2008)

[7] Coclite, M.M., Palmieri, G.: On a singular nonlinear Dirichlet problem. Comm. Partial Differ. Equ. 14, 1315-1327 (1989)

[8] Costa, D.G., Magalhães, C.A.: Existence results for perturbations of the p-Laplacian. Nonlinear Anal. 24, 409-418 (1995)

[9] de Figueiredo, D., Gossez, J.P., Ubilla, P.: Local superlinearity and sublinearity for the $p$-Laplacian. J. Funct. Anal. 257, 721-752 (2009)

[10] de Paiva, F.O., Massa, E.: Multiple solutions for some elliptic equations with a nonlinearity concave at the origin. Nonlinear Anal. 66, 2940-2946 (2007)

[11] Egnell, H.: Elliptic boundary value problems with singular coefficients and critical nonlinearities. Indiana Univ. Math. J. 38, 235-251 (1989)

[12] Fei, G.: On periodic solutions of superquadratic Hamiltonian systems. Electron. J. Differ. Equ. 8, 1-12 (2002)

[13] García Azorero, J., Manfredi, J., Peral Alonso, I.: Sobolev versus Hölder local minimizers and global multiplicity for some quasilinear elliptic equations. Commun. Contemp. Math. 2, 385-404 (2000)

[14] Gasiński, L., Papageorgiou, N.S.: Nonsmooth Critical Point Theory and Nonlinear Boundary Value Problems. Chapman and Hall/ CRC Press, Boca Raton (2005)

[15] Gasiński, L., Papageorgiou, N.S.: Nonlinear Analysis. Chapman and Hall/CRC Press, Boca Raton (2006)

[16] Gasiński, L., Papageorgiou, N.S.: Multiple solutions for noncoercive problems with the p-Laplacian. Bull. Belg. Math. Soc. Simon Stevin 17, 83-99 (2010)

[17] Giacomoni, J., Prashanth, S., Sreenadh, K.: $W^{1, N}$ versus $C^{1}$ local minimizers for elliptic functionals with critical growth in $\mathbb{R}^{N}$. C. R. Math. Acad. Sci. Paris 347, 255-260 (2009)

[18] Giacomoni, J., Saoudi, K.: $W_{0}^{1, p}$ versus $C^{1}$ local minimizers for a singular and critical functional. J. Math. Anal. Appl. 363, 697-710 (2010)

[19] Giacomoni, J., Schindler, I., Takáč, P.: Sobolev versus Hölder local minimizers and existence of multiple solutions for a singular quasilinear equation. Ann. Sc. Norm. Super. Pisa Cl. Sci. 6(5), 117-158 (2007)

[20] Guo, Z., Zhang, Z.: $W^{1, p}$ versus $C^{1}$ local minimizers and multiplicity results for quasilinear elliptic equations. J. Math. Anal. Appl. 286, 32-50 (2003)

[21] Hernández, J., Mancebo, F.J.: Singular elliptic and parabolic equations. In: Handbook of Differential Equations: Stationary Partial Differential Equations, Chap. 4, vol. 3, pp. 317-400. Elsevier (2010) 
[22] Hsu, T.S., Lin, H.L.: Multiple positive solutions for singular elliptic equations with concave-convex nonlinearities and sign-changing weight. Bound. Value Probl. 23, 1-17 (2009)

[23] Lair, A.V., Shaker, A.W.: Classical and weak solutions of a singular semilinear elliptic problems. J. Math. Anal. Appl. 211, 371-385 (1997)

[24] Li, S.-J., Wu, S., Zhou, H.-S.: Solutions to semilinear elliptic problems with combined nonlinearities. J. Differ. Equ. 185, 200-224 (2002)

[25] Motreanu, D., Motreanu, V.V., Papageorgiou, N.S.: A degree theoretic approach for multiple solutions of constant sign for nonlinear elliptic equations. Manuscr. Math. 124, 507-531 (2007)

[26] Perera, K.: Multiplicity result for some elliptic problems with concave nonlinearity. J. Differ. Equ. 140, 133-141 (1997)

[27] Perera, K., Silva, E.A.B.: Existence and multiplicity of positive solutions for singular quasilinear problems. J. Math. Anal. Appl. 323, 1238-1252 (2006)

[28] Perera, K., Zhang, Z.: Multiple positive solutions of singular $p$-Laplacian problems by variational methods. Bound. Value Probl. 3, 377-382 (2005)

[29] Shi, J., Yao, M.: On a singular nonlinear semilinear elliptic problem. Proc. R. Soc. Edinburgh Sect. A 128, 1389-1401 (1998)

[30] Sun, Y., Wu, S., Long, Y.: Combined effects of singular and superlinear nonlinearities in some singular boundary value problems. J. Differ. Equ. 176, 511$531(2001)$

[31] Terracini, S.: On positive entire solutions to a class of equations with a singular coefficient and critical exponent. Adv. Differ. Equ. 1, 241-264 (1996)

[32] Vázquez, J.L.: A strong maximum principle for some quasilinear elliptic equations. Appl. Math. Optim. 12, 191-202 (1984)

[33] Zhang, Z.: Critical points and positive solutions of singular elliptic boundary value problems. J. Math. Anal. Appl. 302, 476-483 (2005)

Leszek Gasiński

Institute of Computer Science

Jagiellonian University

ul. Łojasiewicza 6

30-348 Kraków, Poland

e-mail: Leszek.Gasinski@ii.uj.edu.pl

Nikolaos S. Papageorgiou

Department of Mathematics

National Technical University

Zografou Campus

Athens 15780, Greece

e-mail: npapg@math.ntua.gr

Communicated by Rafael D. Benguria.

Received: June 25, 2010.

Accepted: July 4, 2011. 